\title{
Super- and sub-rotating equatorial jets in shallow water models of Jovian atmospheres: Newtonian cooling versus Rayleigh friction
}

\author{
Emma S. Warneford and Paul J. Dellar $\nmid$ \\ OCIAM, Mathematical Institute, University of Oxford, Andrew Wiles Building, Radcliffe Observatory Quarter, Woodstock Road, \\ Oxford, OX2 6GG, UK
}

(Received 27 August 2013; revised 30 December 2016; accepted 7 April 2017)

\begin{abstract}
Numerical simulations of the shallow water equations on rotating spheres produce mixtures of robust vortices and alternating zonal jets, as seen in the atmospheres of the gas giant planets. However, simulations that include Rayleigh friction invariably produce a sub-rotating (retrograde) equatorial jet for Jovian parameter regimes, whilst observations of Jupiter show a super-rotating (prograde) equatorial jet that has persisted over several decades. Super-rotating equatorial jets have recently been obtained in shallow water simulations that include a Newtonian relaxation of perturbations to the layer thickness to model radiative cooling to space, and in simulations of the thermal shallow water equations that include a similar relaxation term in their temperature equation. Simulations of global quasigeostrophic forms of these different models produce equatorial jets in the same directions as the parent models, suggesting that mechanism responsible for setting the direction lies within quasigeostrophic theory. We provide such a mechanism by calculating the effective force acting on the thickness-weighted zonal mean flow due to the decay of an equatorially trapped Rossby wave. Decay due to Newtonian cooling creates an eastward zonal mean flow at the equator, consistent with the formation of a super-rotating equatorial jet, while decay due to Rayleigh friction leads to a westward zonal mean flow at the equator, consistent with the formation of a sub-rotating equatorial jet. In both cases the meridionally integrated zonal mean of the absolute zonal momentum is westward, consistent with the standard result that Rossby waves carry westward pseudomomentum, but this does not preclude the zonal mean flow being eastward on and close to the equator.
\end{abstract}

\section{Introduction}

Observations of Jupiter's atmosphere reveal a highly turbulent cloud deck containing long-lived coherent vortices, such as the Great Red Spot, that are transported around the planet by an alternating pattern of zonal jets (Vasavada \& Showman 2005). Following the pioneering non-divergent barotropic model of Williams (1978), shallow water theory has been widely used to model the Jovian atmosphere (Cho \& Polvani 1996a,b; Dowling \& Ingersoll 1989; Iacono et al. 1999a,b; Ingersoll 1990; Marcus 1988; Scott \& Polvani 2007, 2008; Showman 2007; Williams \& Yamagata 1984). The visible cloud deck is treated as a homogeneous dynamically active layer separated from a much deeper and relatively quiescent lower layer by a sharp density contrast, which may be linked to latent heat being released at the water vapour condensation level. The equivalent barotropic approximation (Dowling \& Ingersoll 1989; Gill 1982) gives a closed set of shallow water equations for the flow in the cloud deck.

Numerical simulations of the shallow water equations on rotating spheres with Jovian parameter values reproduce a mixture of robust vortices and alternating zonal jets. The latter arise naturally from the nonlinear inverse cascade characteristic of two-dimensional turbulence being arrested through coupling to Rossby waves at the Rhines (1975) scale, though the quasilinear "zonostrophic instability" mechanism may also have a role (Srinivasan \& Young 2012, 2014 , and references therein). The equatorial jets are invariably sub-rotating (retrograde) in both freely decaying and forced-dissipative simulations in the Jovian regime (e.g. Cho \& Polvani 1996a,b; Iacono et al. 1999a,b; Scott \& Polvani 2007; Showman 2007). By contrast, the mean zonal wind profiles in figure 1, as obtained from tracking visible features in Jupiter's atmosphere, show a broad, super-rotating equatorial jet. The similarity of the two profiles, taken from the Voyager 2 (Limaye 1986) and Cassini (Porco et al. 2003) missions over 20 years apart, demonstrates the remarkable stability of Jupiter's zonal winds, and the longevity of the super-rotating equatorial jet.

Forced-dissipative shallow water simulations typically include a Rayleigh friction term to absorb the slow inverse cascade of energy past the Rhines scale to the largest scales in the system, which tends to create large coherent vortices in place of the alternating zonal jets (e.g. Vasavada \& Showman 2005). Scott \& Polvani $(2007,2008)$ added an additional radiative relaxation term with timescale $\tau_{\text {rad }}$ to the continuity equation in their shallow water model:

$$
\begin{aligned}
h_{t}+\boldsymbol{\nabla} \cdot(h \boldsymbol{u}) & =-\left(h-h_{0}\right) / \tau_{\text {rad }}, \\
\boldsymbol{u}_{t}+(\boldsymbol{u} \cdot \boldsymbol{\nabla}) \boldsymbol{u}+f \hat{\boldsymbol{z}} \times \boldsymbol{u} & =-g^{\prime} \boldsymbol{\nabla} h+\boldsymbol{F}-\boldsymbol{u} / \tau_{\text {fric }},
\end{aligned}
$$

Here $h$ is the thickness of the active layer, commonly called "height", $\boldsymbol{u}$ is the depth-averaged horizontal velocity, $f=2 \Omega \sin \phi$ is the Coriolis parameter at latitude $\phi$ on a planet rotating with angular velocity $\Omega, \nabla$ is the horizontal 


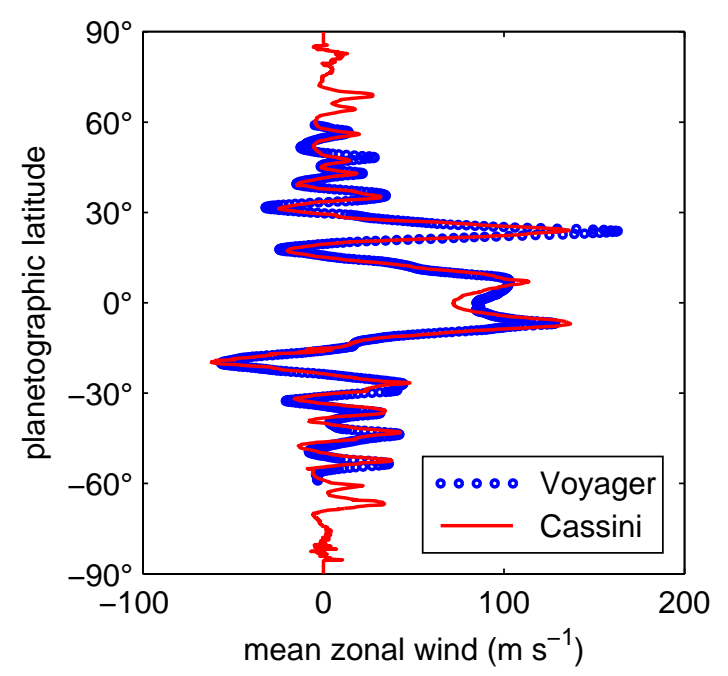

FIGURE 1. Observed mean zonal winds versus planetographic latitude for Jupiter. The blue dots show Voyager 2 data from Limaye (1986) and the red line shows Cassini data from Porco et al. (2003).

gradient operator, $\hat{z}$ is a unit vector in the local vertical direction, $\boldsymbol{F}$ is an isotropic random forcing, and $\tau_{\text {fric }}$ is the timescale for Rayleigh friction. The reduced gravity is $g^{\prime}=g \Delta \rho / \rho_{0}$, where $g$ is the actual gravitational acceleration, $\Delta \rho$ is the density contrast between the cloud deck and the lower layer, and $\rho_{0}$ is the density of the lower layer. These parameters are possibly constrained by observations of what appear to be internal gravity waves radiating from the impact points of Shoemaker-Levy comet debris (Dowling 1995; Ingersoll et al. 2007), although this interpretation is not without its critics (Walterscheid et al. 2000).

The right hand side of $(1.1 a)$ models the effects of radiation to space with a Newtonian relaxation of $h$ towards its constant mean value $h_{0}$ on the time scale $\tau_{\text {rad }}$, following earlier shallow water models of the upper ocean mixed layer (Hirst 1986; Philander et al. 1984) and the terrestrial stratosphere (Juckes 1989; Polvani et al. 1995; Thuburn \& Lagneau 1999). Scott \& Polvani's $(2007 ; 2008)$ simulations of this system with dissipation only by thickness relaxation (no $\tau_{\text {fric }}$ term) produced a sharply localised super-rotating equatorial jet, as do our subsequent simulations described in $\S 3$, and in Warneford \& Dellar (2014).

Thermal shallow water theory was introduced by Lavoie (1972) to describe atmospheric mixed layers over frozen lakes. It permits horizontal variations of the thermodynamic properties of the fluid within each layer, which are taken to be uniform in the standard shallow water equations. The density contrast $\Delta \rho$ thus becomes a dynamical variable. However, subsequent developments of thermal shallow water theory have been much more focused on modelling the oceanic mixed layer (Hirst 1986; McCreary et al. 1991; McCreary \& Kundu 1988; McCreary \& Yu 1992; Ripa 1993, 1995, 1996a,b; Røed \& Shi 1999; Schopf \& Cane 1983).

Warneford \& Dellar (2014) simulated Jovian atmospheres using the forced-dissipative thermal shallow water equations

$$
\begin{aligned}
h_{t}+\nabla \cdot(h \boldsymbol{u}) & =0 \\
\Theta_{t}+(\boldsymbol{u} \cdot \nabla) \Theta & =-\left(\Theta h / h_{0}-\Theta_{0}\right) / \tau_{\mathrm{rad}}, \\
\boldsymbol{u}_{t}+(\boldsymbol{u} \cdot \nabla) \boldsymbol{u}+f \hat{\boldsymbol{z}} \times \boldsymbol{u} & =-h^{-1} \nabla\left(\frac{1}{2} \Theta h^{2}\right)+\boldsymbol{F}-\boldsymbol{u} / \tau_{\text {fric }} .
\end{aligned}
$$

We introduce the symbol $\Theta=g \Delta \rho / \rho_{0}$ for the reduced gravity to emphasise that it is now a function of space and time. The first term on the right hand side of $(1.2 c)$ is then the usual shallow water pressure gradient term, as rewritten for a spatially varying reduced gravity. The right hand side of $(1.2 b)$ is a Newtonian cooling term that represents radiative relaxation towards a temperature $\Theta_{0} h_{0} / h$ with time scale $\tau_{\text {rad }}$. We show below that this form of coupling between $\Theta$ and $h$ changes the behaviour of equatorially trapped Rossby waves in almost exactly the same way as the radiative relaxation of $h$ itself in Scott \& Polvani's $(2007 ; 2008)$ model. In particular, the damping rates calculated in $\S 4$ are very similar function of wavenumber and $\tau_{\mathrm{rad}}$. The resulting Reynold stresses calculated in $\S 5$ have very similar spatial structures between the two models, and are proportional to the difference between the dimensionless radiative and frictional decay rate constants in both models.

The thermal shallow water equations $(1.2 a, b, c)$ conserve both mass and momentum in the absence of the $\tau_{\text {fric }}$ and $\boldsymbol{F}$ terms, unlike the Scott and Polvani model $(1.1 a, b)$. Simulations of the thermal shallow water equations for Jovian parameter values (reported in $\S 3$ and Warneford \& Dellar 2014) reproduce a super-rotating equatorial jet, and more substantial mid-latitude jets than simulations of the Scott \& Polvani $(2007,2008)$ model. Henceforth, for brevity we refer to the radiative relaxation terms as relaxation and the Rayleigh friction term as friction. We also refer to equations $(1.1 a, b)$ as the "standard" shallow water equations to distinguish them from the thermal shallow water equations $(1.2 a, b, c)$.

Quasi-geostrophic (QG) theory (Charney 1949; Obukhov 1949) offers a simplified description of slow vortical mo- 
tions that filters out inertia-gravity waves. Although QG theory is usually employed for mid-latitude beta-planes, Matsuno $(1970,1971)$ presented a global QG theory for fully stratified atmospheres on spheres. Verkley (2009) and Schubert et al. (2009) revived this theory for the standard shallow water equations on a sphere in the form

$$
q_{t}+[\psi, q]=0, \quad q=f+\nabla^{2} \psi-L_{\mathrm{D}}^{-2} \psi,
$$

where the Jacobian $[\psi, q]=\hat{\boldsymbol{z}} \cdot(\boldsymbol{\nabla} \psi \times \boldsymbol{\nabla} q)$. The Rossby deformation scale is $L_{\mathrm{D}}=c / f$, where $c$ is the speed of short shallow water gravity waves. Both $f$ and $L_{\mathrm{D}}$ vary with latitude when QG theory is used in spherical coordinates. The sole evolving variable is the potential vorticity $q$, which is advected by the velocity field derived from a streamfunction $\psi$. The elliptic equation relating $\psi$ to $q$ involves the spatially varying Coriolis parameter $f$. It corresponds to Daley's (1983) simplest form of geostrophic balance, as justified by assuming that $\psi$ varies on lengthscales much smaller than the planetary scales on which $f$ varies. The system (1.3) reduces to the familiar beta-plane QG equations on approximating $f$ by $f_{0}+\beta y$ in the first term, and by $f_{0}$ in the second term. Warneford (2014) derived a thermal form of this global QG theory, building on the beta-plane thermal QG equations in Ripa (1996a) and Warneford \& Dellar (2013). Numerical simulations produced super-rotating equatorial jets when the dimensionless radiative relaxation time $\tau_{\text {rad }}$ is sufficiently short, as did simulations of the corresponding global QG form of Scott \& Polvani's $(2007 ; 2008)$ shallow water model with a relaxing thickness. By contrast, both QG models produced sub-rotating equatorial jets when $\tau_{\text {rad }}$ is sufficiently long. The mechanism responsible for setting the equatorial jet direction must therefore lie within QG theory. This focuses attention on momentum fluxes due to Rossby waves, rather than by inertia-gravity waves, or the equatorially trapped Kelvin and Yanai waves. None of these waves exist in QG theory.

\section{Momentum transport by Rossby waves}

Dickinson (1969) showed that the meridional flux of zonal momentum due to Rossby waves produces a net eastward acceleration in regions where Rossby waves are generated, and a net westward acceleration in regions where Rossby waves are dissipated. This argument was further developed by Green (1970) and Thompson (1971). A detailed version based on ray theory may be found in Held (2000) and Vallis (2006). The QG equations on a mid-latitude beta plane lead to the Rossby wave dispersion relation $\omega=-\beta k /\left(k^{2}+\ell^{2}+L_{\mathrm{D}}^{2}\right)$ for plane-wave disturbances with streamfunction $\psi=\hat{\psi} \exp (\mathrm{i}(k x+\ell y-\omega t))$ implies a meridional group velocity

$$
\frac{\partial \omega}{\partial \ell}=\frac{2 \beta k \ell}{\left(k^{2}+\ell^{2}+L_{\mathrm{D}}^{2}\right)^{2}},
$$

while the off-diagonal component of the Reynolds stress (see $\S 4$ for notation) is

$$
\left\langle u^{\prime} v^{\prime}\right\rangle=-\frac{1}{2} \hat{\psi}^{2} k \ell
$$

The radiation condition requires the meridional group velocity to point away from wave sources, so the sign of $k \ell$ is such as to create a flux of westward (negative) zonal momentum away from sources of Rossby waves towards regions where Rossby waves are dissipated. For example, Schneider \& Liu (2009) and Liu \& Schneider (2010) identified Rossby wave sources due to divergent motions associated with the breakdown of geostrophy near the equator in their threedimensional simulations of Jovian atmospheres. Propagation of these Rossby waves to higher latitudes thus creates a net eastward acceleration at the equator according to the above theory, which is consistent with the formation of superrotating equatorial jets in their simulations.

However, the focus of our present paper will be on the equatorially trapped Rossby waves that play a key role in nearequatorial dynamics (e.g. Gill 1982; Khouider et al. 2013; Majda \& Klein 2003; Matsuno 1966; McCreary 1985). These waves are confined by a Gaussian envelope whose extent scales with the equatorial deformation scale $L_{\mathrm{eq}}=\sqrt{c /(2 \beta)}$. This corresponds to latitudes within about $30^{\circ}$ of the equator for Jovian parameters (see $\S 4$ ). We therefore need to compute the latitude-dependence of the momentum fluxes inside the scale of this envelope, for which a ray theory approach is inadequate.

The generalised Lagrangian mean (GLM) theory is a powerful approach for addressing this and other problems in wave-mean flow interaction (Andrews \& McIntyre 1976a; Bühler 2000, 2014). It defines the Lagrangian mean of an arbitrary field $\phi(\boldsymbol{x}, t)$ by

$$
\phi^{\mathrm{L}}(\boldsymbol{x}, t)=\overline{\phi(\boldsymbol{x}+\boldsymbol{\xi}(\boldsymbol{x}, t), t)},
$$

where $\overline{(\cdots)}$ is some linear averaging operator, and $\boldsymbol{\xi}(\boldsymbol{x}, t)$ is a Lagrangian displacement field such that $\boldsymbol{x}+\boldsymbol{\xi}(\boldsymbol{x}, t)$ is the actual position of the particle whose mean position is $\boldsymbol{x}$ at time t. GLM theory establishes circumstances for the validity of a "pseudomomentum rule" (Bühler 2000, 2014; McIntyre 1981) under which the waves behave for some purposes as if they carried a momentum equal to their pseudomomentum and the background fluid were absent. The pseudomomentum is a disturbance quantity related to the pseudoenergy defined in $\S 5$.

A linearized version of GLM for small $\boldsymbol{\xi}$ was presented in Andrews \& McIntyre $(1976 a, 1978)$. However, the Lagrangian picture of a fluid evolving through displacements of indestructible particles fits awkwardly with the mass source or sink term on the right hand side of $(1.1 a)$ that models radiative relaxation. In particular, lack of mass conservation 


$\begin{array}{cccccc}a & 2 \pi / \Omega & \sqrt{g^{\prime} h_{0}} & g & \tau_{\text {rad }} & \tau_{\text {fric }} \\ 7.1 \times 10^{7} \mathrm{~m} & 9.9 \text { hours } & 678 \mathrm{~m} \mathrm{~s}^{-1} & 26 \mathrm{~m} \mathrm{~s}^{-2} & 429 \text { hours } & 9900 \text { hours }\end{array}$

TABLE 1. Parameter values for Jupiter (from Beebe 1994; Ingersoll 1990; Warneford \& Dellar 2014).

requires modification of Bühler's (2000) shallow water pseudomomentum rule obtained from GLM theory. Nevertheless, McIntyre (2014, personal communication) has established, firstly, that is possible to reproduce our results below using the linearised GLM theory slightly adapted to apply to $(1.1 a, b)$ and, secondly, that an eastward zonal acceleration at the equator is obtained for two interesting cases, (1) equatorially trapped Rossby waves freely decaying by radiative relaxation - as we compute independently below - and (2) the same waves, but maintained at constant amplitude by a balance between forcing and dissipation, with the dissipation again by radiative relaxation but with the forcing mimicked by negative Rayleigh friction. The linearised GLM theory shows why negative Rayleigh friction, if spatially uniform, has an effect qualitatively similar to that of free temporal decay - as illustrated by the curves in figure $4 \mathrm{~b}$ of Andrews \& McIntyre (1976a) with suitable sign changes.

Following the approach of McIntyre \& Norton (1990) for stratified fluids, Bühler (2000) formulated a general approach for shallow water equations of the form

$$
\begin{aligned}
h_{t}+\nabla \cdot(h \boldsymbol{u}) & =0, \\
\boldsymbol{u}_{t}+(\boldsymbol{u} \cdot \nabla) \boldsymbol{u}+f \hat{\boldsymbol{z}} \times \boldsymbol{u} & =-g^{\prime} \boldsymbol{\nabla} h+\boldsymbol{F}
\end{aligned}
$$

with a general term $\mathbf{F}$, not necessarily a forcing. The shallow water potential vorticity $q=(f+\zeta) / h$, where $\zeta=$ $\hat{z} \cdot(\nabla \times \boldsymbol{u})$ is the relative vorticity, evolves according to

$$
\partial_{t}(h q)+\nabla \cdot\left(h q \boldsymbol{u}-\boldsymbol{F}^{\perp}\right)=0,
$$

with an extra flux $\boldsymbol{F}^{\perp}=\hat{\boldsymbol{z}} \times \boldsymbol{F}$. Bühler's (2000) pseudomomentum rule holds for systems of this form that conserve linear momentum, i.e. those for which $h \boldsymbol{F}=\nabla \cdot \boldsymbol{\sigma}$ is the divergence of a stress tensor $\boldsymbol{\sigma}$

However, neither of of the shallow water models from $\S 1$ fits this form. The shallow water system with relaxation of the thickness does not conserve momentum. In the absence of forcing and friction, $(1.1 a)$ and (1.1b) together imply

$$
(h \boldsymbol{u})_{t}+\nabla \cdot\left(h \boldsymbol{u} \boldsymbol{u}+\frac{1}{2} g^{\prime} h^{2} \boldsymbol{I}\right)+f \hat{\boldsymbol{z}} \times(h \boldsymbol{u})=-\left(h-h_{0}\right) \boldsymbol{u} / \tau_{\mathrm{rad}}
$$

where $\boldsymbol{I}$ is the $2 \times 2$ identity matrix. Moreoever, the potential vorticity for the system $(1.1 a, b)$ obeys

$$
(h q)_{t}+\nabla \cdot\left(h q u+\boldsymbol{u}^{\perp} / \tau_{\text {fric }}\right)=0,
$$

where $\boldsymbol{u}^{\perp}=\hat{\boldsymbol{z}} \times \boldsymbol{u}$, while the potential vorticity in the thermal shallow water equations $(1.2 a-c)$ obeys

$$
(h q)_{t}+\nabla \cdot\left(h q u-\frac{1}{2} h \nabla^{\perp} \theta+\boldsymbol{u}^{\perp} / \tau_{\text {fric }}\right)=0 .
$$

Radiative relaxation thus does not contribute to either potential vorticity equation. However, (2.8) contains an extra term involving the perpendicular temperature gradient $\nabla^{\perp} \theta=\hat{z} \times \nabla \theta$ that arises from the curl of the $(1 / 2) h \boldsymbol{\nabla} \Theta$ term on the right hand side of $(1.2 c)$. The thermal shallow water equations thus do not materially conserve potential vorticity, even in the absence of forcing and dissipation. Instead, the total potential vorticity inside a closed isotherm is conserved. This is the thermal shallow water analogue of the potential vorticity impermeability property of isentropic surfaces in three-dimensional stratified fluids (Haynes \& McIntyre 1990).

Since the Scott \& Polvani (2007, 2008) radiatively relaxed shallow water model lacks both mass and momentum conservation, and hence does not fit naturally into the above general frameworks, in $\S 4$ and $\S 5$ below we derive from first principles equations for the evolution of the thickness-weighted zonal mean velocity components $\langle u\rangle^{*}$ and $\langle v\rangle^{*}$ (see $\S 5$ for notation) caused by the decay of an equatorially trapped Rossby wave by friction and/or radiative relaxation.

\section{Numerical experiments}

We now show some numerical simulations of the standard shallow water equations with dissipation only through friction (i.e. $(1.1 a, b)$ with no $\tau_{\text {rad }}$ term), the Scott \& Polvani $(2007,2008)$ model with relaxation of the thickness, and our thermal shallow water model. Table 1 gives typical Jovian values for the planetary radius $a$, angular velocity $\Omega$, gravitational acceleration $g$, and internal wave speed $\sqrt{g^{\prime} h_{0}}$. The last is deduced from impacts of Shoemaker-Levy comet debris (Dowling 1995; Ingersoll et al. 2007), and implies that there are 232 polar deformation scales $L_{\mathrm{D}}=$ $\sqrt{g^{\prime} h_{0}} /(2 \Omega)$ around the circumference. We used a radiative relaxation timescale $\tau_{\text {rad }}$ of 43 Jovian days, as calculated for a temperature of $120 \mathrm{~K}$ at the 25 millibar pressure level, and all simulations employed a friction with a time scale of 1000 Jovian days. Liu \& Schneider (2010) used the 25 millibar pressure level when comparing their three-dimensional simulations with observations of Neptune. Calculating $\tau_{\text {rad }}$ at the 25 millibar pressure level for both planets leads to a super-rotating equatorial jet for Jupiter, and a sub-rotating equatorial jet for Neptune, in our thermal shallow water 


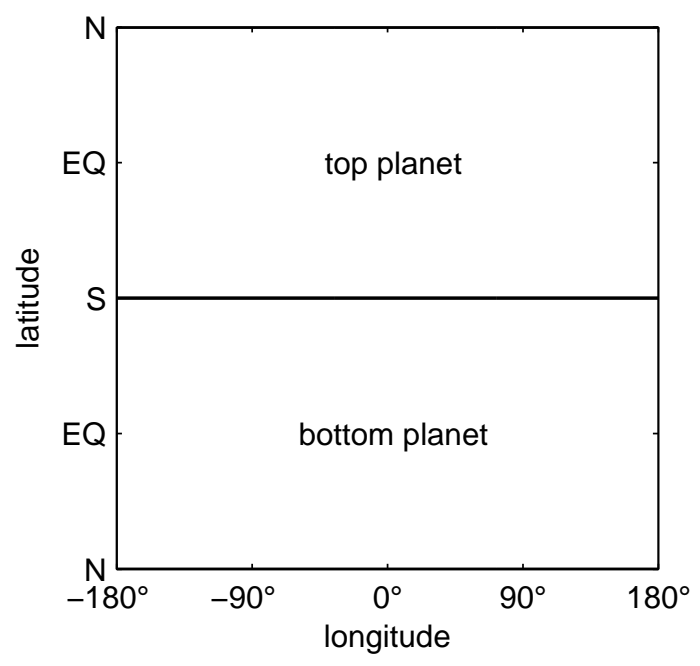

FIGURE 2. Schematic of the doubly-periodic Cartesian domain used for the numerical simulations. Each half of the domain represents a full planet, so for ease of interpretation we show simulation results for just the top planet.

model (Warneford \& Dellar 2014). The theory in $\S 5$ below suggests that the direction of the equatorial jet is insensitive to these parameters provived the radiative relaxation timescale remains much shorter than the frictional timescale.

Simulations of geostrophic turbulence in the Jovian regime require thousands of rotation periods to reach statistically steady states. The superior memory bandwidth and peak floating point performance of graphical processing units (GPUs) thus becomes attractive, but existing spherical harmonic transform algorithms for GPUs only achieve performance parity with conventional microprocessors (Hupca et al. 2012). We therefore employed the doubly-periodic Cartesian domain sketched in figure 2, which we refer to as the square planet domain. The horizontal axis denotes longitude, while the vertical axis denotes latitude. Starting from the north pole at the top of the domain and moving down, we reach the equator a quarter of the way down, and the south pole half way down. We then continue to reach the equator again, before returning to the north pole at the bottom of the domain. We imagine following a meridian (constant longitude line) from the north pole to the equator to the south pole, and then following a second meridian with longitude offset by $180^{\circ}$ back across the equator to the north pole. The Coriolis force is still fully varying in latitude, and is defined in the simulations by $f(y)=2 \Omega \cos \left(2 \pi y / y_{\max }\right)$, where $y_{\max }$ is the length of the side of the square planet domain, i.e. the circumference of Jupiter (see table 1). The Coriolis force and all its derivatives are thus doubly periodic. This allowed us to exploit a high performance GPU fast Fourier transform library. We discretized the domain using $1024 \times 1024$ Fourier collocation points, and used the Hou \& Li (2007) spectral filter to control the build-up of enstrophy at the highest wavenumbers. We integrated the resulting large system of ordinary differential equations using the standard fourth-order Runge-Kutta scheme, with a time step determined dynamically from the Courant-Friedrichs-Lewy stability condition.

Our initial conditions were $h=h_{0}$ and $u=v=0$ for all runs, and $\Theta=\Theta_{0}$ for the thermal shallow water simulation. Following Scott \& Polvani (2007, 2008) we applied a divergence-free isotropic random forcing $\boldsymbol{F}$ that is localised to a narrow annulus of wavenumbers $|\mathbf{k}| \in[40,44]$ in Fourier space, counting the longest axis-aligned sinusoidal mode in the domain as wavenumber 1 . We forced each mode inside this annulus with amplitude $\epsilon_{f}$ using random phases that were $\delta$-correlated (white) in time. This forcing is widely used in numerical studies of zonal jet formation, and models the energy injected by three-dimensional convection at horizontal lengthscales comparable to the deformation radius. Our simulations slowly adjusted the amplitude of the forcing $\epsilon_{f}$ to reach a prescribed value of the total kinetic energy in the eventual statistical steady state. Further details of the numerical models, parameters, and simulation outputs may be found in Warneford (2014) and Warneford \& Dellar (2014).

Figure 3 shows the instantaneous absolute vorticity $\omega_{\mathrm{a}}=v_{x}-u_{y}+f(y)$ after $2 \times 10^{4}$ Jovian days for the top planet in figure 2 for three different simulations: the standard shallow water equations with friction alone, the standard shallow water equations with friction and radiative relaxation of the thickness, and the thermal shallow water equations with friction and radiative relaxation of the temperature. Figure 4 shows the corresponding instantaneous zonal velocity plots, and figure 5 shows the instantaneous zonally averaged zonal velocity, $\langle u\rangle=y_{\max }^{-1} \int u(x, y, t) \mathrm{d} x$. All three simulations produced a mixture of vortices, turbulence and multiple zonal jets, with amplitudes that decrease at higher latitudes. The two simulations with radiative relaxation produced a strong super-rotating equatorial jet in line with observations of Jupiter. However, our simulation of the standard shallow water model, with dissipation only by friction, produced a subrotating equatorial jet. The simulation with radiative relaxation of the thickness produced very weak jets away from the equator, whereas the other two simulations produced stronger mid-latitude jets in better agreement with observations. 


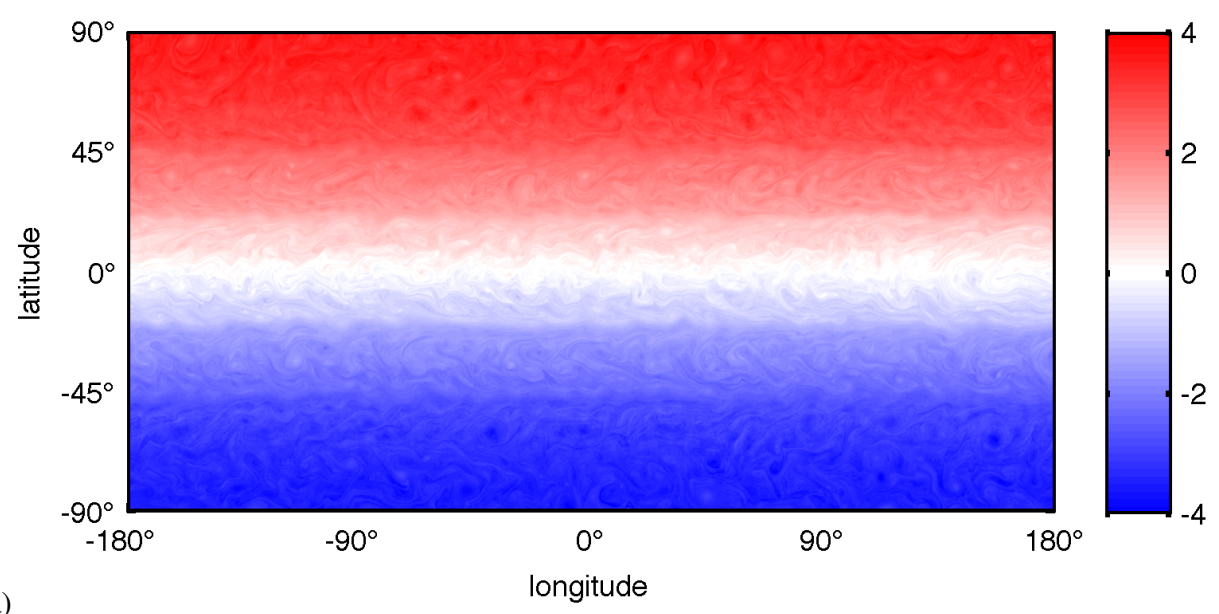

(a)

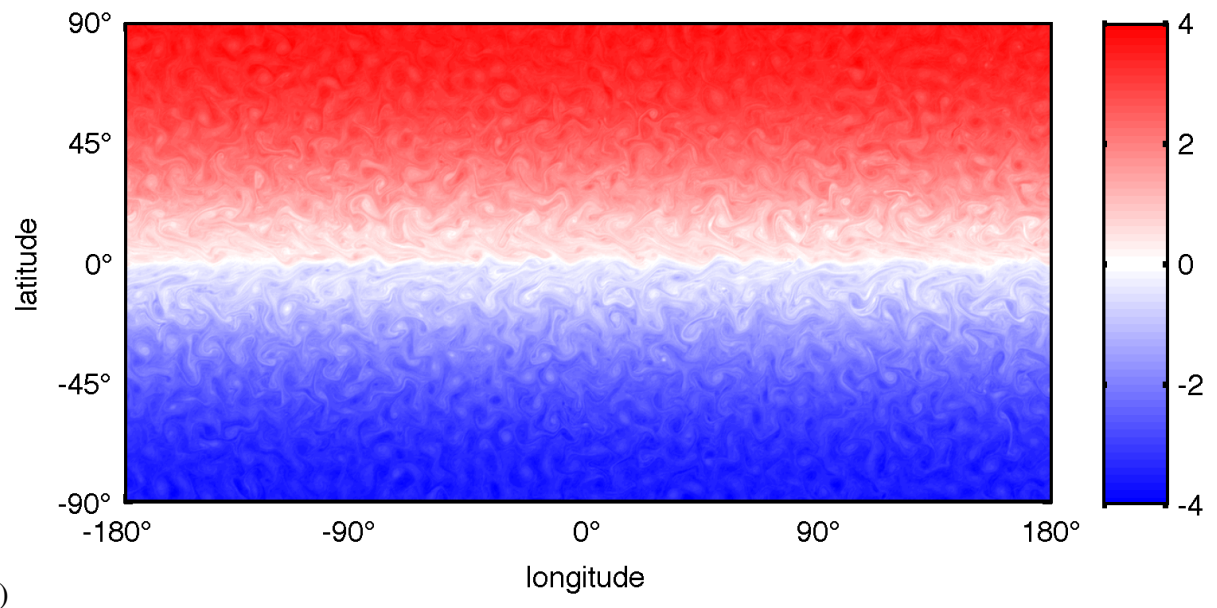

(b)

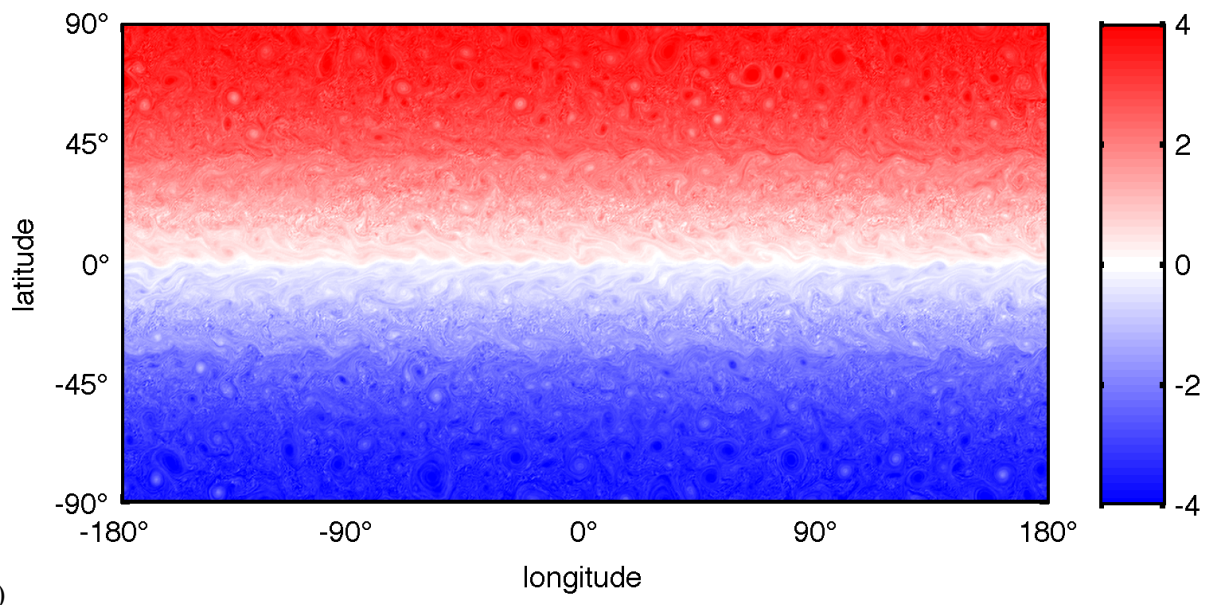

FIGURE 3. Absolute vorticity $\omega_{\mathrm{a}}=v_{x}-u_{y}+f(y)$ in units of $10^{-4} \mathrm{~s}^{-1}$ for (a) the standard shallow water equations with dissipation by friction alone, (b) the standard shallow water equations with friction and radiative relaxation of the thickness, and (c) the thermal shallow water equations with friction and radiative relaxation of the temperature.

\section{Rossby waves on an equatorial beta-plane}

We now investigate the properties of equatorially trapped waves on an equatorial beta plane in the different shallow water models, as they decay freely due to Rayleigh friction and/or Newtonian radiative relaxation. The equatorial beta plane uses local Cartesian coordinates with $x$ eastward and $y$ northward. The underlying spherical geometry appears only through the linearisation of $f=2 \Omega \sin \phi$ for small latitudes $|\phi| \ll 1$ into $f(y)=\beta y$ with $\beta=2 \Omega / a$, where $a$ is the planetary radius. 


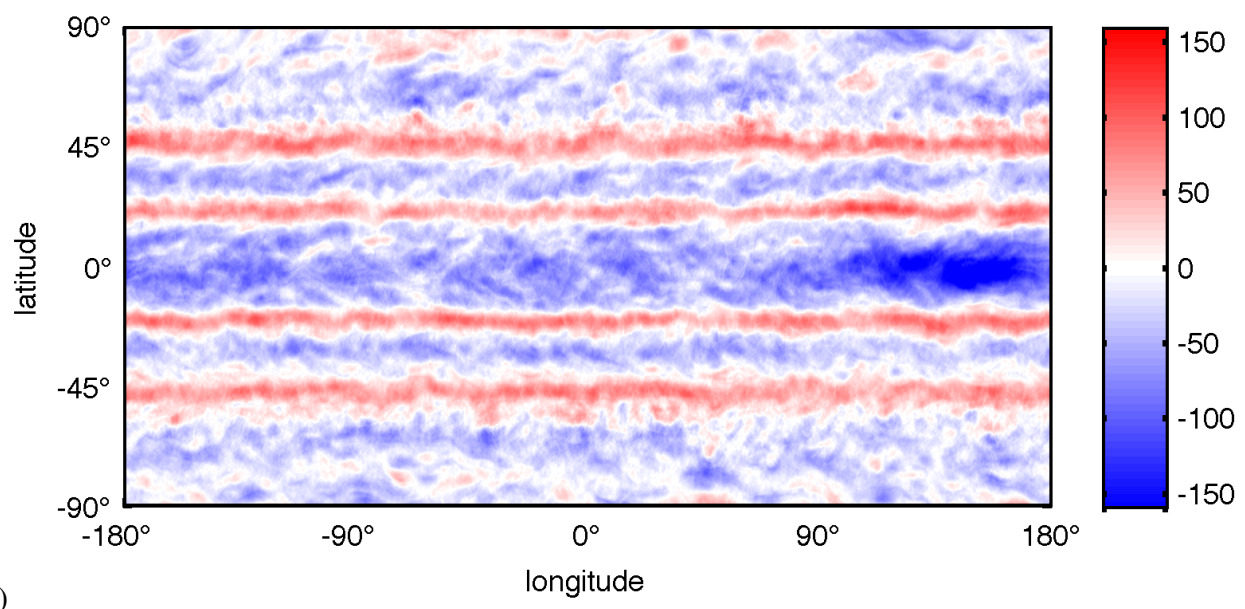

(a)

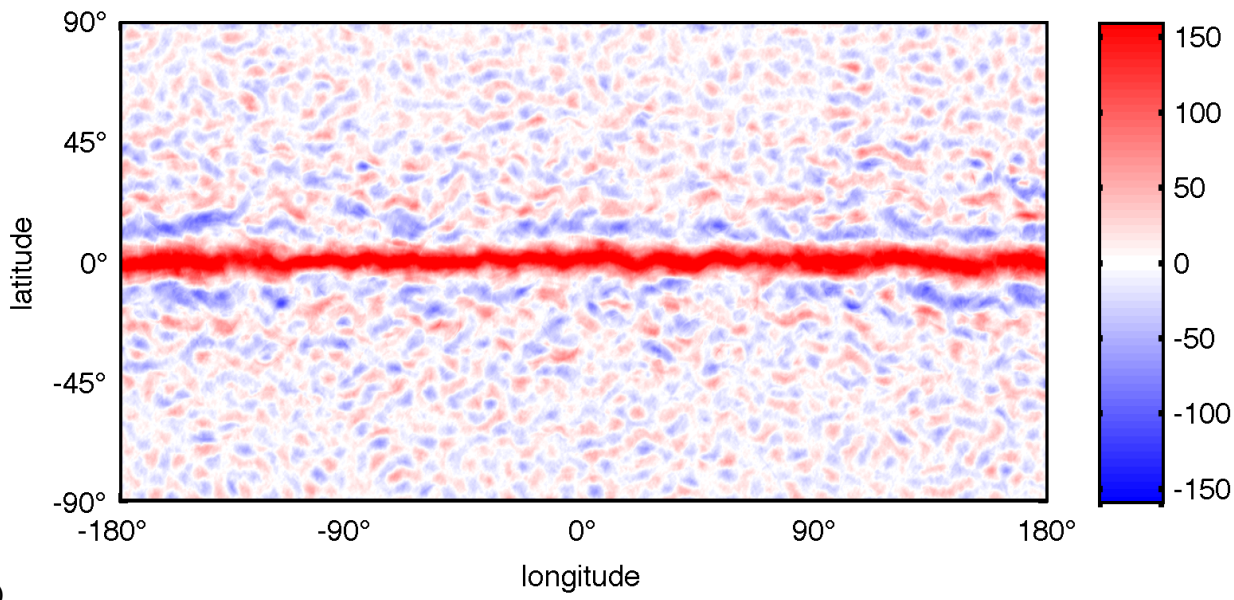

(b)

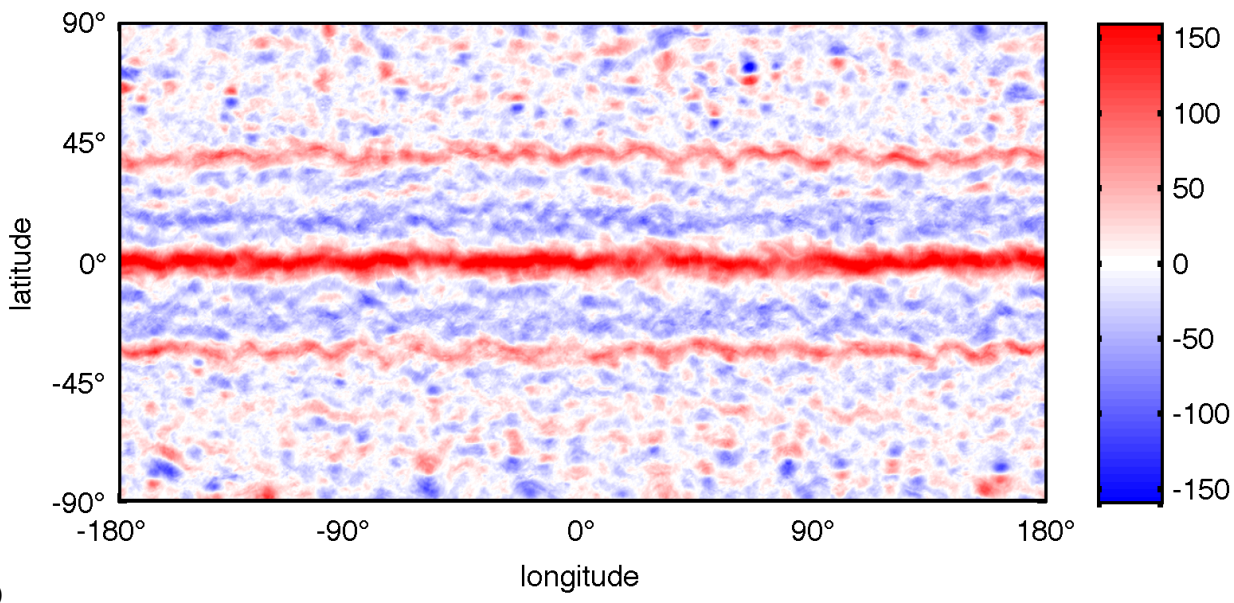

FIGURE 4. Zonal velocity $u$ in units of $\mathrm{m} \mathrm{s}^{-1}$ for (a) the standard shallow water equations with dissipation by friction alone, (b) the standard shallow water equations with friction and radiative relaxation of the thickness, and (c) the thermal shallow water equations with friction and radiative relaxation of the temperature.

\subsection{Shallow water equations with thickness relaxation}

The linearised form of the unforced $(\boldsymbol{F}=\mathbf{0})$ shallow water equations (1.1) with radiative relaxation of the thickness on an equatorial beta-plane may be written as (Gill 1982; Matsuno 1966)

$$
\begin{aligned}
h_{t}^{\prime}+u_{x}^{\prime}+v_{y}^{\prime} & =-\kappa h^{\prime}, \\
u_{t}^{\prime}-\frac{1}{2} y v^{\prime}+h_{x}^{\prime} & =-\gamma u^{\prime}, \\
v_{t}^{\prime}+\frac{1}{2} y u^{\prime}+h_{y}^{\prime} & =-\gamma v^{\prime} .
\end{aligned}
$$

The dashes denote small perturbations about a rest state with uniform depth $h_{0}$. We have non-dimensionalised using the internal wave speed $c=\sqrt{g^{\prime} h_{0}}$ as the velocity scale, the equatorial deformation radius $L_{\mathrm{eq}}=\sqrt{c / 2 \beta}$ as the horizontal 


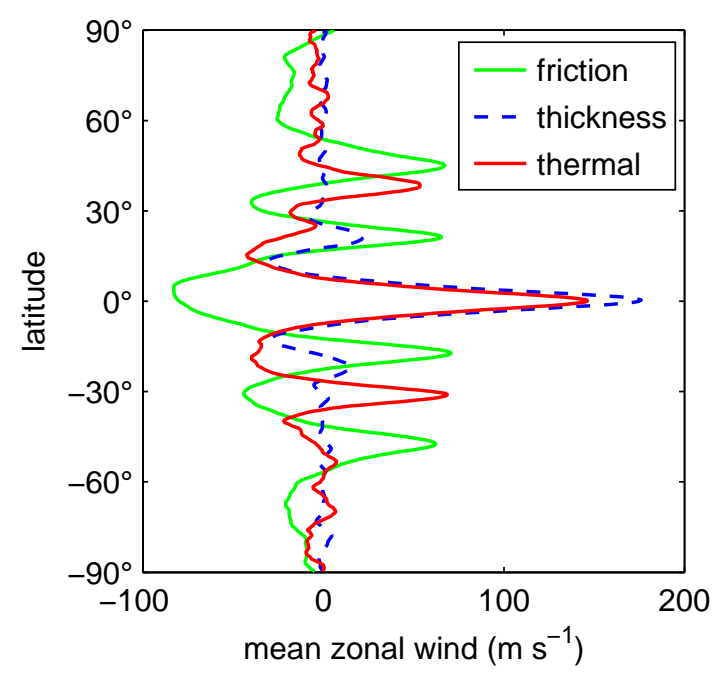

FIGURE 5. Mean zonal velocity $\langle u\rangle$ against latitude for simulations of the standard shallow water equations with dissipation by friction alone, the standard shallow water equations with friction and radiative relaxation of the thickness, and the thermal shallow water equations with friction and radiative relaxation of the temperature

length scale, and $h_{0}$ as the thickness scale. Using the Jovian parameters in table 1 , a latitude of $33^{\circ}$ corresponds to $y=5$ in the subsequent plots. The dimensionless radiative relaxation and friction rates are $\kappa=T / \tau_{\text {rad }}$ and $\gamma=T / \tau_{\text {fric }}$ based on the advective time scale $T=L_{\text {eq }} / c$. Their values were $\kappa=7.88 \times 10^{-3}$ and $\gamma=T / \tau_{\text {fric }}=3.42 \times 10^{-4}$ in our simulation with radiative relaxation of the thickness.

Following standard practice (e.g. Gill 1982; Matsuno 1966) we seek waves that are harmonic in longitude and time of the form $h^{\prime}(x, y, t)=\operatorname{Re}\{\hat{h}(y) \exp (\mathrm{i}(k x-\omega t))\}$ etc. With no dissipation $(\kappa=\gamma=0)$ the three equations (4.1) may be combined into a single ordinary differential equation (ODE) for the meridional velocity $\hat{v}(y)$,

$$
\frac{\mathrm{d}^{2} \hat{v}}{\mathrm{~d} y^{2}}=\left(A y^{2}-B\right) \hat{v}, \quad A=\frac{1}{4}, \quad B=\omega^{2}-k^{2}-\frac{k}{2 \omega},
$$

the same equation that governs a quantum harmonic oscillator. The solutions that decay as $y \rightarrow \pm \infty$ may be written using the Hermite polynomials $H_{n}(\xi)$ as

$$
\hat{v}=H_{n}(\xi) \exp \left(-\xi^{2} / 2\right), \quad \xi=y A^{1 / 4} .
$$

The dispersion relation $A^{-1 / 2} B=2 n+1$ for $n=-1,0,1,2, \ldots$ gives a cubic equation for $\omega$,

$$
\omega^{2}-k^{2}-\frac{k}{2 \omega}=n+\frac{1}{2}
$$

The Rossby wave branch of solutions is characterised by $n \geqslant 1$ and $0<-\omega / k \ll 1$. The other two roots give inertiagravity waves with $|\omega| \geqslant 1$ (the dimensionless inertial frequency). The cases $n=0$ and $n=-1$ give the Yanai and Kelvin waves respectively. Figure 6 shows these different branches of the dispersion relation, all of which represent trapped waves localised within a few equatorial deformation scales $L_{\text {eq }}$ of the equator. The inertia-gravity, Kelvin, and Yanai wave branches all exceed the inertial frequency $|\omega|=1$. The inertia-gravity and Kelvin waves thus do not exist in QG theory, while the QG Yanai wave's frequency approaches 0 as $k \rightarrow 0$ like a Rossby wave. The mechanism responsible for setting the direction of equatorial jet in the shallow water models is expected to lie within QG theory, so we only consider the Rossby waves in the remainder of this paper.

The friction and radiative relaxation terms in $(4.1 a-c)$ change the $A$ and $B$ coefficients to (Yamagata $\&$ Philander 1985)

$$
A=\frac{1}{4}\left(\frac{\omega+\mathrm{i} \kappa}{\omega+\mathrm{i} \gamma}\right), \quad B=\omega^{2}-k^{2}-\frac{k}{2(\omega+\mathrm{i} \gamma)}+\mathrm{i} \omega(\kappa+\gamma)-\kappa \gamma
$$

so the dispersion relation becomes

$$
\omega^{2}-k^{2}-\frac{k}{2(\omega+\mathrm{i} \gamma)}+\mathrm{i} \omega(\kappa+\gamma)-\kappa \gamma=\left(n+\frac{1}{2}\right)\left(\frac{\omega+\mathrm{i} \kappa}{\omega+\mathrm{i} \gamma}\right)^{1 / 2} \text { for } n=1,2, \ldots
$$

Figure 7 shows the real and imaginary parts of the complex frequency $\omega$ for the first few equatorial Rossby waves ( $n=1,2,3)$ for different values of $\gamma$ and $\kappa$. The dispersion relation (4.4) is invariant under the transformation $(k, \omega) \mapsto$ $(-k,-\omega)$, so we choose to take $k>0$. The $\operatorname{Rossby}$ wave frequency $\operatorname{Re}(\omega)$ is now negative, and virtually unchanged by either weak friction $(\kappa=0.001, \gamma=0)$ or weak radiative relaxation $(\kappa=0, \gamma=0.001)$. The decay rate $-\operatorname{Im}(\omega) \operatorname{due}$ to cooling $(\kappa=0.001, \gamma=0)$ is largest at $k=0$, and tends to zero as $k \rightarrow \infty$. By contrast, the decay due to friction 


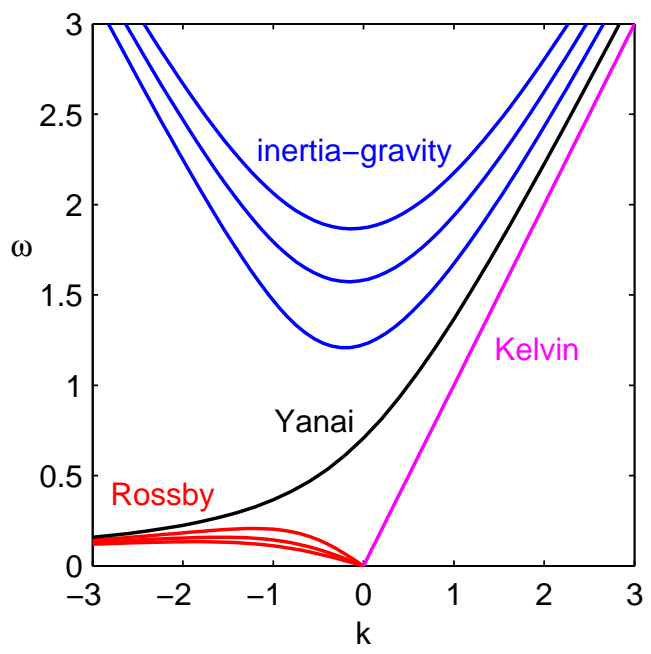

FIGURE 6. Dimensionless dispersion relation (4.4) for equatorially trapped waves in the standard shallow water equations on the equatorial beta-plane with no dissipation.
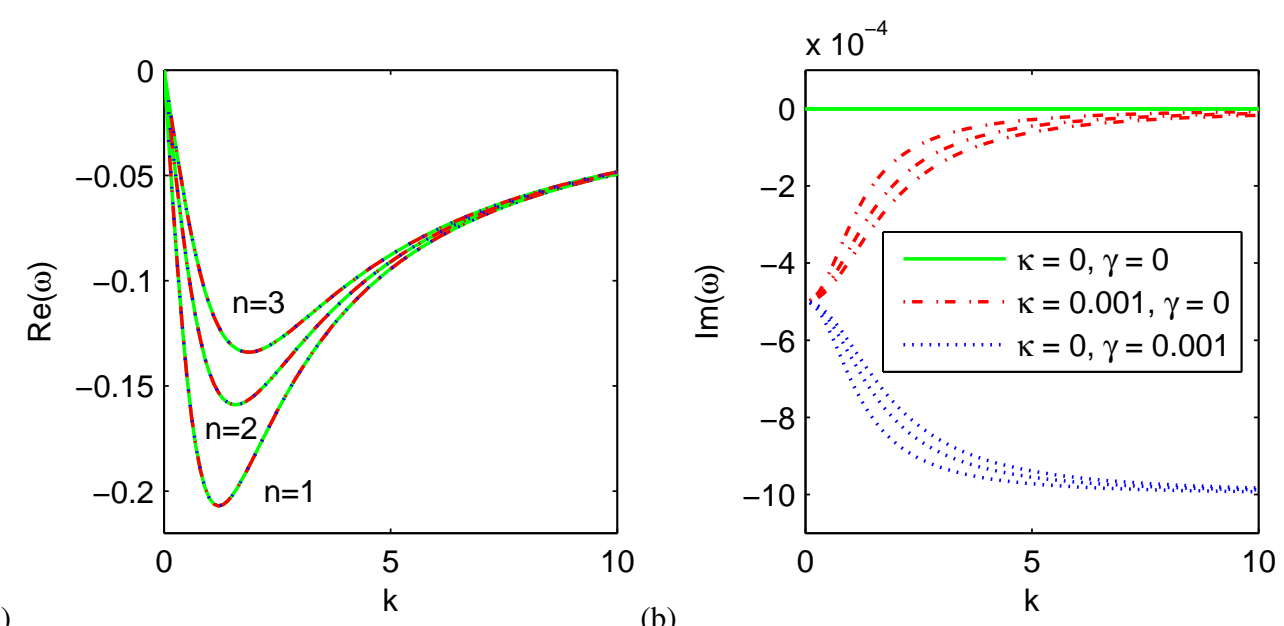

FIGURE 7. The (a) real and (b) imaginary parts of the dimensionless equatorial Rossby wave frequency $\omega$ for the standard shallow water equations with no dissipation (green solid lines), dissipation by relaxation of the thickness (red dash-dot lines), and dissipation by friction (blue dotted lines). The three lines appear coincident in panel (a).

( $\gamma=0.001, \kappa=0$ ) is monotonically increasing with $k$, and $-\operatorname{Im}(\omega)$ tends to $-\gamma$ as $k \rightarrow \infty$. Figure $7 \mathrm{~b}$ also suggests a symmetry about the line $\operatorname{Im}(\omega)=-\epsilon / 2$ between small radiative relaxation $(\kappa=\epsilon$ and $\gamma=0)$ and small friction $(\kappa=0$ and $\gamma=\epsilon)$. We expand the roots of (4.6) as $\omega=\omega_{0}+\epsilon \omega_{1, \text { rad }}+\epsilon^{2} \omega_{2, \text { rad }}+\cdots$ for radiative relaxation, and $\omega=\omega_{0}+\epsilon \omega_{1, \text { fric }}+\epsilon^{2} \omega_{2, \text { fric }}+\cdots$ for friction, where $\omega_{0}$ is the real root of (4.4). The $O(\epsilon)$ corrections are both purely imaginary:

$$
\begin{aligned}
& \omega_{1, \text { rad }}=\frac{1}{2} \mathrm{i} \omega_{0}\left(-4 \omega_{0}^{2}+2 n+1\right) /\left(k+4 \omega_{0}^{3}\right), \\
& \omega_{1, \text { rric }}=\frac{1}{2} \mathrm{i} \omega_{0}\left(-4 \omega_{0}^{2}-2 n-1-2 k / \omega_{0}\right) /\left(k+4 \omega_{0}^{3}\right) .
\end{aligned}
$$

The symmetry about $\operatorname{Im}(\omega)=-\epsilon / 2$ visible in figure $7 \mathrm{~b}$ is a consequence of the relation

$$
\omega_{1, \text { rad }}+\frac{\mathrm{i}}{2}=\frac{\mathrm{i}}{2}\left(\frac{k+(2 n+1) \omega_{0}}{k+4 \omega_{0}^{3}}\right)=-\left(\omega_{1, \text { fric }}+\frac{\mathrm{i}}{2}\right)
$$

between the two dispersion relations truncated at $O(\epsilon)$.

\subsection{Thermal shallow water equations}

Linearising the thermal shallow water equations $(1.2 a-c)$ about a rest state with uniform depth $h_{0}$ and temperature $\Theta_{0}$, and applying the equivalent nondimensionalisation to $\S 4.1$ based on the velocity scale $\sqrt{h_{0} \Theta_{0}}$ gives

$$
\begin{aligned}
h_{t}^{\prime}+u_{x}^{\prime}+v_{y}^{\prime} & =0, \\
\Theta_{t}^{\prime} & =-\kappa\left(h^{\prime}+\Theta^{\prime}\right), \\
u_{t}^{\prime}-\frac{1}{2} y v^{\prime}+h_{x}^{\prime}+\frac{1}{2} \Theta_{x}^{\prime} & =-\gamma u^{\prime} \\
v_{t}^{\prime}+\frac{1}{2} y u^{\prime}+h_{y}^{\prime}+\frac{1}{2} \Theta_{y}^{\prime} & =-\gamma v^{\prime} .
\end{aligned}
$$




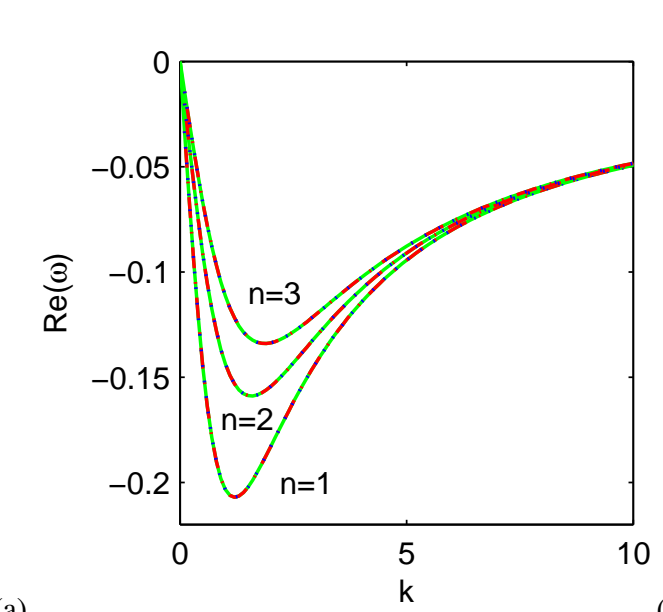

(a)

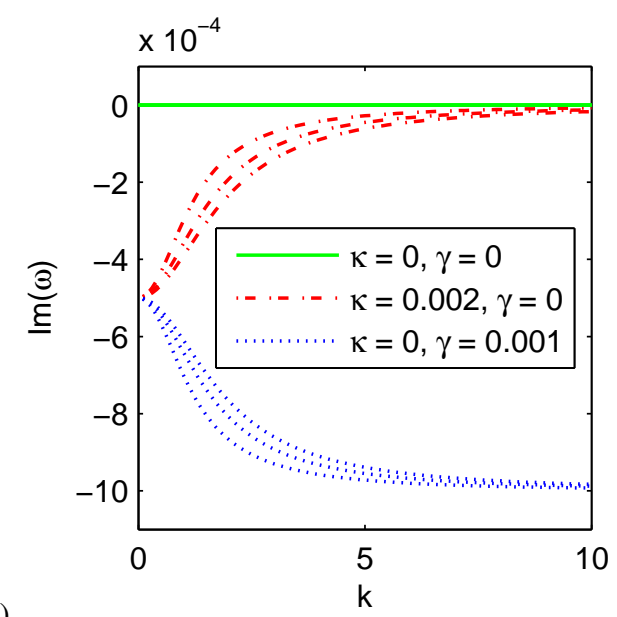

(b)

FIGURE 8 . The real and imaginary parts of the dimensionless equatorial Rossby wave frequency $\omega$ for the thermal shallow water equations with no dissipation (green solid lines), dissipation by relaxation of the temperature (red dash-dot lines), and dissipation by friction (blue dotted lines). The three lines appear coincident in panel (a).

The four equations (4.9) may again be combined into the single ODE (4.2) for $\hat{v}(y)$, with coefficients

$$
A=\frac{1}{4} \frac{\omega(\omega+\mathrm{i} \kappa)}{(\omega+\mathrm{i} \gamma)(\omega+\mathrm{i} \kappa / 2)}, \quad B=\frac{\omega(\omega+\mathrm{i} \gamma)(\omega+\mathrm{i} \kappa)}{\omega+\mathrm{i} \kappa / 2}-k^{2}-\frac{k}{2(\omega+\mathrm{i} \gamma)},
$$

so the dispersion relation for equatorially trapped waves is

$$
\frac{\omega(\omega+\mathrm{i} \gamma)(\omega+\mathrm{i} \kappa)}{\omega+\mathrm{i} \kappa / 2}-k^{2}-\frac{k}{2(\omega+\mathrm{i} \gamma)}=\left(n+\frac{1}{2}\right)\left(\frac{\omega(\omega+\mathrm{i} \kappa)}{(\omega+\mathrm{i} \gamma)(\omega+\mathrm{i} \kappa / 2)}\right)^{1 / 2} .
$$

Figure 8 shows the real and imaginary parts of the complex frequency $\omega$ for the first few equatorial Rossby waves with $n \in\{1,2,3\}$, and three different sets of $\kappa$ and $\gamma$ values. As before, the real part of $\omega$ is virtually unchanged by weak dissipation $(\kappa=0.002$ or $\gamma=0.001)$ while $\operatorname{Im}(\omega)$ becomes negative. The plot of $\operatorname{Im}(\omega)$ in figure 8 closely resembles our earlier figure 7, with the same apparent symmetry, if we now take $\kappa=2 \gamma$. A similar perturbation analysis to before shows that including this factor of 2 indeed makes $\operatorname{Im}(\omega)$ symmetric about $-\epsilon / 2$ between the cases of small radiative relaxation $(\kappa=2 \epsilon$ and $\gamma=0)$ and small friction $(\kappa=0$ and $\gamma=\epsilon)$.

\section{Acceleration of the zonal mean zonal flow}

We now investigate the acceleration of the zonal mean zonal flow created by these solutions for decaying equatorially trapped Rossby waves.

\subsection{Shallow water equations with thickness relaxation}

We consider the shallow water model (1.1) with radiative relaxation of the thickness, and no forcing, on an equatorial beta-plane, and apply the nondimensionalisation from $\S 4.1$ to obtain

$$
\begin{aligned}
h_{t}+(h u)_{x}+(h v)_{y} & =-\kappa(h-1), \\
(h u)_{t}+\left(h u^{2}+\frac{1}{2} h^{2}\right)_{x}+(h u v)_{y}-\frac{1}{2} y h v & =-\gamma h u-\kappa u(h-1), \\
(h v)_{t}+(h u v)_{x}+\left(h v^{2}+\frac{1}{2} h^{2}\right)_{y}+\frac{1}{2} y h u & =-\gamma h v-\kappa v(h-1) .
\end{aligned}
$$

The mean thickness $h_{0}$ becomes unity under this nondimensionalisation.

Following standard practice, we decompose the velocity and other fields as $\boldsymbol{u}=\langle\boldsymbol{u}\rangle+\boldsymbol{u}^{\prime}$ into a zonal mean $\langle\boldsymbol{u}\rangle$ and a deviation $\boldsymbol{u}^{\prime}$. The zonal mean of the dimensionless mass conservation equation may then be written as

$$
\langle h\rangle_{t}+\langle h v\rangle_{y}=-\kappa(\langle h\rangle-1) .
$$

Similarly, the zonal means of the two momentum equations are

$$
\langle h u\rangle_{t}+\langle h u v\rangle_{y}-\frac{1}{2} y\langle h v\rangle=-\gamma\langle h u\rangle-\kappa(\langle h u\rangle-\langle u\rangle),
$$

and

$$
\langle h v\rangle_{t}+\left\langle h v^{2}+\frac{1}{2} h^{2}\right\rangle_{y}+\frac{1}{2} y\langle h u\rangle=-\gamma\langle h v\rangle-\kappa(\langle h v\rangle-\langle v\rangle) .
$$

We now consider the form of solutions to the system $(5.1 a, b, c)$ for initial conditions corresponding to a Rossby wave of small amplitude $\mathcal{O}(a)$ superimposed on a rest state with unit thickness. For these initial conditions, and under the 
subsequent evolution, the thickness and velocity components satisfy the scalings

$$
h=1+\underbrace{h^{\prime}}_{\mathcal{O}(a)}+\underbrace{(\langle h\rangle-1)}_{\mathcal{O}\left(a^{2}\right)}, \quad u=\underbrace{u^{\prime}}_{\mathcal{O}(a)}+\underbrace{\langle u\rangle}_{\mathcal{O}\left(a^{2}\right)}, \quad v=\underbrace{v^{\prime}}_{\mathcal{O}(a)}+\underbrace{\langle v\rangle}_{\mathcal{O}\left(a^{2}\right)} .
$$

We define $\langle\eta\rangle^{*}=\langle h \eta\rangle /\langle h\rangle$ to be the thickness-weighted zonal mean of a quantity $\eta$, as in Thuburn \& Lagneau (1999). Using $\langle h\rangle=1+\mathcal{O}\left(a^{2}\right)$, we can simplify the above to obtain evolution equations correct to $\mathcal{O}\left(a^{2}\right)$ in the form

$$
\begin{aligned}
\langle h\rangle_{t}+\langle v\rangle_{y}^{*}+\kappa(\langle h\rangle-1) & =0, \\
\langle u\rangle_{t}^{*}-\frac{1}{2} y\langle v\rangle^{*}+\gamma\langle u\rangle^{*} & =-\left\langle u^{\prime} v^{\prime}\right\rangle_{y}-\kappa\left\langle h^{\prime} u^{\prime}\right\rangle \\
\langle v\rangle_{t}^{*}+\frac{1}{2} y\langle u\rangle^{*}+\gamma\langle v\rangle^{*}+\langle h\rangle_{y} & =-\left\langle v^{\prime} v^{\prime}\right\rangle_{y}-\kappa\left\langle h^{\prime} v^{\prime}\right\rangle-\frac{1}{2}\left\langle h^{\prime 2}\right\rangle_{y} .
\end{aligned}
$$

On the left hand sides we have only the three mean quantities $\langle h\rangle,\langle u\rangle^{*},\langle v\rangle^{*}$. On the right hand sides we have only means of quadratic products of the fluctuations $h^{\prime}, u^{\prime}, v^{\prime}$. These include the meridional derivatives of the Reynolds stress components $\left\langle u^{\prime} v^{\prime}\right\rangle$ and $\left\langle v^{\prime} v^{\prime}\right\rangle$. The contribution $-\frac{1}{2}\left\langle h^{\prime 2}\right\rangle_{y}$ from the fluctuating pressure gradient arises from using (5.5) to write

$$
h^{2}=1+2 h^{\prime}+2(\langle h\rangle-1)+h^{\prime 2}+\mathcal{O}\left(a^{3}\right) .
$$

There are also radiative relaxation terms proportional to the so-called bolus velocity components $\left\langle h^{\prime} u^{\prime}\right\rangle$ and $\left\langle h^{\prime} v^{\prime}\right\rangle$. These terms would be absent for the momentum-conserving variant of the shallow water equations with relaxation of the thickness.

The effect of the Rossby wave thus appears as an effective force on the right hand sides of the evolution equations for $\langle u\rangle^{*}$ and $\langle v\rangle^{*}$. Our use of the thickness-weighted means $\langle u\rangle^{*}$ and $\langle v\rangle^{*}$ in place of the unweighted means $\langle u\rangle$ and $\langle v\rangle$ eliminates a $\left\langle h^{\prime} v^{\prime}\right\rangle$ term that would otherwise appear in (5.6a). This is similar to the transformed Eulerian mean (TEM) approach for stratified fluids (Andrews \& McIntyre 1976a, 1978).

A further simplification is possible by assuming that geostrophic balance holds in the meridional momentum equation (5.6c), since the meridional lengthscale and velocity component are both smaller than the zonal lengthscale and zonal velocity component. The same scaling argument is used in the semigeostrophic theory of atmospheric front formation. The time derivative of this meridional geostrophic balance condition gives

$$
\frac{1}{2} y\langle u\rangle_{t}^{*}+\langle h\rangle_{y t}=-\frac{1}{2}\left\langle h^{\prime 2}\right\rangle_{y t},
$$

which can be used in place of (5.6c) to obtain a closed system with (5.6a) and (5.6b).

We now evaluate these right hand sides using the equatorially trapped Rossby wave solutions in $\S 4.1$. These represent small perturbations about a state of rest with unit depth in dimensionless variables. The meridional velocity perturbation is $v^{\prime}=\alpha \operatorname{Re}\{\hat{v}(y) \exp (\mathrm{i}(k x-\omega t))\}$, with $\hat{v}(y)$ defined by (4.3) and (4.5), and the constant $\alpha$ sets the amplitude. The corresponding zonal velocity and thickness perturbations are $u^{\prime}=\alpha \operatorname{Re}\{\hat{u}(y) \exp (\mathrm{i}(k x-\omega t))\}$ and $h^{\prime}=\alpha \operatorname{Re}\{\hat{h}(y) \exp (\mathrm{i}(k x-\omega t))\}$, where

$$
\hat{u}(y)=\mathrm{i}\left(\frac{k(\mathrm{~d} \hat{v} / \mathrm{d} y)-\frac{1}{2} y(\omega+\mathrm{i} \kappa) \hat{v}}{k^{2}-(\omega+\mathrm{i} \gamma)(\omega+\mathrm{i} \kappa)}\right), \quad \hat{h}(y)=-\mathrm{i}\left(\frac{(\mathrm{i} \omega-\gamma) \hat{u}+\frac{1}{2} y \hat{v}}{k}\right),
$$

for $k \neq 0$. The denominator in the expression for $\hat{u}(y)$ vanishes when $k^{2}=(\omega+\mathrm{i} \gamma)(\omega+\mathrm{i} \kappa)$. This gives the dispersion relation for equatorially trapped Kelvin waves, which are distinguished by having zero meridional velocity $(\hat{v}=0)$, and frequencies $\omega= \pm k$ in the absence of dissipation.

To allow a fair comparison between the effective forces generated by waves with different $n$ and $k$ values, we normalise the disturbance amplitudes by choosing $\alpha$ so that

$$
\frac{1}{2} \int\left\langle u^{\prime 2}\right\rangle+\left\langle v^{\prime 2}\right\rangle+\left\langle h^{\prime 2}\right\rangle \mathrm{d} y=a^{2} .
$$

The left hand side of (5.10) is the quadratic approximation to the disturbance pseudoenergy, an exact conserved quantity of the unforced, non-dissipative shallow water equations that takes the above form when expanded for small-amplitude disturbances from a rest state of constant depth (Shepherd 1993). Using a standard formula for the average of a product of sinusoidal disturbances represented by the real parts of complex exponentials, we can compute the integrand in (5.10), and the terms on the right hand sides of $(5.6 b)$ and $(5.6 c)$, using

$$
\left\langle u^{\prime} v^{\prime}\right\rangle=\frac{1}{2} \operatorname{Re}\left(\hat{u}(y) \hat{v}(y)^{\dagger}\right)
$$

where the superscript ${ }^{\dagger}$ denotes a complex conjugate, and similarly for the other quadratic combinations of $h^{\prime}, u^{\prime}, v^{\prime}$.

Figures 9 and 10 show the two quantities $-\left\langle u^{\prime} v^{\prime}\right\rangle_{y}$ and $-\kappa\left\langle h^{\prime} u^{\prime}\right\rangle$ on the right hand side of (5.6b), and their sum, for waves with $k=1$ and $n \in\{1,2\}$ for dissipation by either Rayleigh friction $(\gamma>0, \kappa=0)$ or radiative relaxation $(\gamma=0, \kappa>0)$. The plots show the quantities divided by $a^{2}$, or as given by setting $a=1$. The Reynolds stress $\left\langle u^{\prime} v^{\prime}\right\rangle$ vanishes for undamped waves, since $\omega, A$, and $\hat{v}$ are then all real, while $\hat{u}$ and $\hat{h}$ are purely imaginary. The bolus velocity term $-\kappa\left\langle h^{\prime} u^{\prime}\right\rangle$ is explicitly proportional to $\kappa$, and so vanishes for $\kappa=0$. 
A perturbative analysis similar to that in $\S 4.1$ shows that the Reynolds stress $\left\langle u^{\prime} v^{\prime}\right\rangle$ is proportional to $\kappa-\gamma$ for small $\kappa$ and $\gamma$. This explains the antisymmetry visible when comparing panel (a) with $\kappa>0, \gamma=0$ panel (b) with $\kappa=0$, $\gamma>0$ in each of figures 9 and 10. Increasing $k$ while holding $n, \kappa, \gamma$ fixed increases the magnitude of $\left\langle u^{\prime} v^{\prime}\right\rangle$ without changing its general shape, as does increasing $\kappa$ or $\gamma$ while holding $k$ and $n$ fixed.

The Reynolds stress convergence is close to zero at the equator when $n$ is odd, leaving just the contribution from the bolus velocity visible in figure 9(a). However, the Reynolds stress convergence has a local extremum on the equator when $n$ is even. Its sign for $n=2$ is such as to produce eastward acceleration at the equator under radiative relaxation (figure 10(a)), and an westward acceleration at the equator under Rayleigh friction (figure 10(b)). We can infer the sign of the acceleration directly from the right hand side of $(5.6 b)$ on the equator. Since $y=0$, the Coriolis contribution from the mean meridional velocity $\langle v\rangle^{*}$ does not contribute to the left hand side of $(5.6 b)$. This leaves the ordinary differential equation

$$
\left.\frac{\mathrm{d}}{\mathrm{d} t}\langle u\rangle^{*}\right|_{y=0}+\left.\gamma\langle u\rangle^{*}\right|_{y=0}=-\left.\mathrm{e}^{-\sigma t}\left(\left\langle u^{\prime} v^{\prime}\right\rangle_{y}+\kappa\left\langle h^{\prime} u^{\prime}\right\rangle\right)\right|_{y=0, t=0}
$$

where $\sigma=-2 \operatorname{Im} \omega$ is twice the decay rate of the Rossby wave calculated in $\S 4$.1. The quadratic products on the right hand side of (5.6b) thus decay in proportion to $\exp (-\sigma t)$. The solution of (5.12) for $\gamma \neq \sigma$ is

$$
\left.\langle u\rangle^{*}\right|_{y=0}=\left.\left[\frac{\mathrm{e}^{-\gamma t}-\mathrm{e}^{-\sigma t}}{\sigma-\gamma}\right]\left(\left\langle u^{\prime} v^{\prime}\right\rangle_{y}+\kappa\left\langle h^{\prime} u^{\prime}\right\rangle\right)\right|_{y=0, t=0}
$$

The time-dependent coefficient in square brackets $[\cdots]$ is always positive for $t>0$, so the direction of the zonal mean flow $\langle u\rangle^{*}$ at the equator is set by the sign of the combined Reynolds stress and bolus velocity term evaluated at $y=0$ and $t=0$. This is consistent with the directions of the equatorial jets found in numerical simulations for different rates of Rayleigh friction and radiative relaxation (Scott \& Polvani 2007, 2008; Warneford \& Dellar 2014).

However, away from the equator one would need to compute the solution to the full response problem coupling $\langle h\rangle$, $\langle u\rangle^{*},\langle v\rangle^{*}$ as three functions of $y$ and $t$ to find the mean flow produced by the wave sources on the right hand sides of (5.6b) and (5.6c). Figure 11 shows the three terms $-\frac{1}{2}\left\langle h^{\prime 2}\right\rangle_{y},-\left\langle v^{\prime} v^{\prime}\right\rangle_{y}$ and $-\kappa\left\langle h^{\prime} u^{\prime}\right\rangle$ on the right hand side of (5.6c), and their sum, for $k=1, n \in\{1,2\}$ and dissipation solely by radiative relaxation $(\kappa=0.001, \gamma=0)$. The first two terms are non-zero even with no dissipation, and typically much larger in magntitude than either the bolus terms or the Reynolds stress convergence in the zonal equation.

Omitting the $\kappa$ terms from the right hand sides of $(5.1 b)$ and $(5.1 c)$ leads to a shallow water model that relaxes the thickeness field but conserves momentum. This model is no less justified than the model that supposes that $\kappa$ should not appear in the equation $(1.1 b)$ for evolving the velocity $\boldsymbol{u}$. The contributions $\left\langle h^{\prime} u^{\prime}\right\rangle$ and $\left\langle h^{\prime} v^{\prime}\right\rangle$ from the bolus velocity would then be absent in the right hand sides of the analogues of (5.6b) and (5.6c) for this model. Figures 9(a) and 10(a) show that these contributions are in any case small compared with the contribution from the Reynolds stress convergence. This is consistent with the directions of the equatorial jets in the two models, with and without momentum conservation, showing the same behaviour in simulations (Warneford \& Dellar 2014).

\subsection{Thermal shallow water equations}

We now apply the same approach to the dimensionless form of the unforced thermal shallow water equations $(1.2 a, b, c, d)$ on an equatorial beta plane. The mass and zonal momentum equations are

$$
\langle h\rangle_{t}+\langle v\rangle_{y}^{*}=0
$$

and

$$
\langle u\rangle_{t}^{*}-\frac{1}{2} y\langle v\rangle^{*}+\gamma\langle u\rangle^{*}=-\left\langle u^{\prime} v^{\prime}\right\rangle_{y}
$$

There are no $\kappa$ terms in these equations, as radiative relaxation conserves mass and momentum in our thermal shallow water model. The temperature $\Theta$ also does not appear in these equations, because the zonal pressure gradient disappears under zonal averaging.

To calculate the thermal contributions to the other equations, it is convenient to decompose the temperature as

$$
\Theta=1+\theta=1+\underbrace{\theta^{\prime}}_{\mathcal{O}(a)}+\underbrace{\langle\theta\rangle}_{\mathcal{O}\left(a^{2}\right)} .
$$

This decomposition eliminates $\mathcal{O}(1)$ terms proportional to the reference temperature $\Theta_{0}$, which is scaled to unity by the nondimensionalisation in $\S 4.2$. Although $\theta^{\prime}=\Theta^{\prime}$, we use $\theta^{\prime}$ below so that all equations are written using the symbol $\theta$.

The meridional momentum equation is

$$
(h v)_{t}+(h u v)_{x}+\left(h v^{2}+\frac{1}{2} h^{2}(1+\theta)\right)_{y}+\frac{1}{2} y h u=-\gamma h v,
$$

and the conservation form of the temperature equation is

$$
(h \theta)_{t}+(h u \theta)_{x}+(h v \theta)_{y}=-\kappa\left(h^{2}(1+\theta)-h\right) .
$$

The unit term from the decomposition $\Theta=1+\theta$ disappears from the left hand side of (5.18) because $h$ satisfies the 


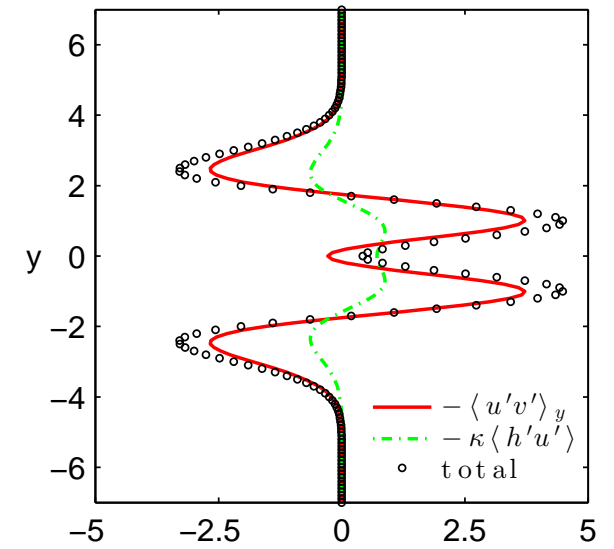

(a)

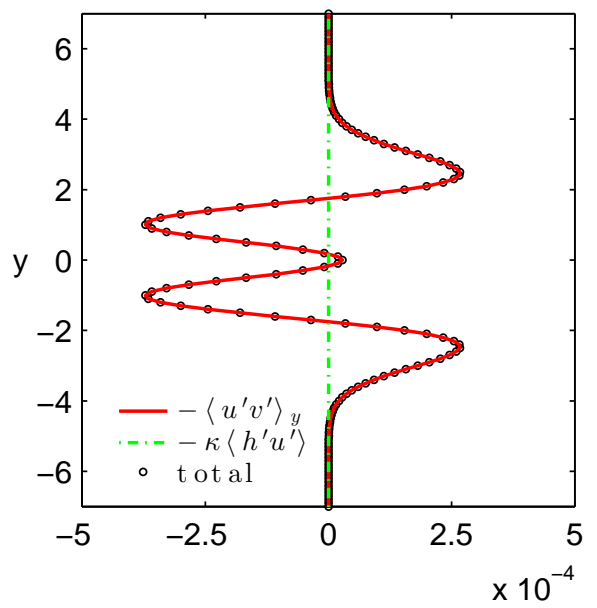

FIGURE 9. The effective zonal forcing terms $-\left\langle u^{\prime} v^{\prime}\right\rangle_{y}$ and $-\kappa\left\langle h^{\prime} u^{\prime}\right\rangle$ on the right hand side of (5.6b), and their sum, for (a) dissipation solely by radiative relaxation $(\kappa=0.001, \gamma=0)$ and (b) dissipation solely by friction $(\kappa=0, \gamma=0.001)$. All terms are calculated for $n=1, k=1$, and $a=1$ for scaling. In panel (b) the sum equals the first term as $\kappa=0$.

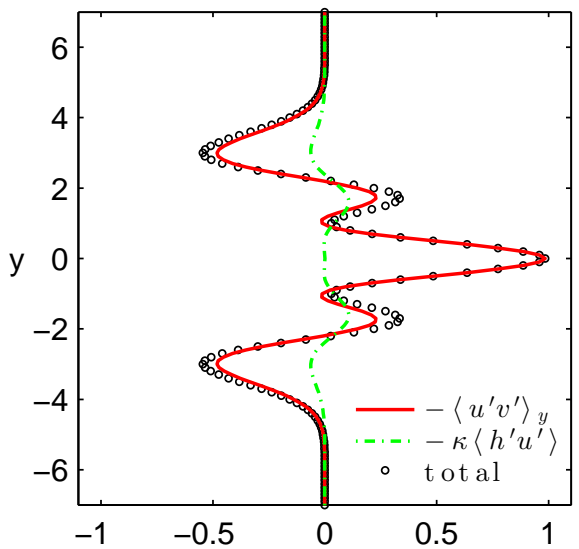

(a) $\times 10^{-3}$ (b)

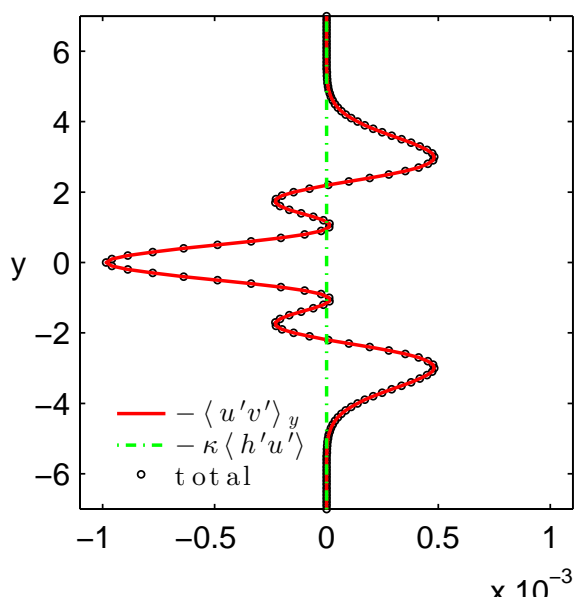

FIgURE 10. The effective zonal forcing terms $-\left\langle u^{\prime} v^{\prime}\right\rangle_{y}$ and $-\kappa\left\langle h^{\prime} u^{\prime}\right\rangle$ on the right hand side of (5.6b), and their sum, for (a) dissipation solely by radiative relaxation $(\kappa=0.001, \gamma=0)$ and $(\mathrm{b})$ dissipation solely by friction $(\kappa=0, \gamma=0.001)$. All terms are calculated for $n=2, k=1$, and $a=1$ for scaling. In panel (b) the sum equals the first term as $\kappa=0$.
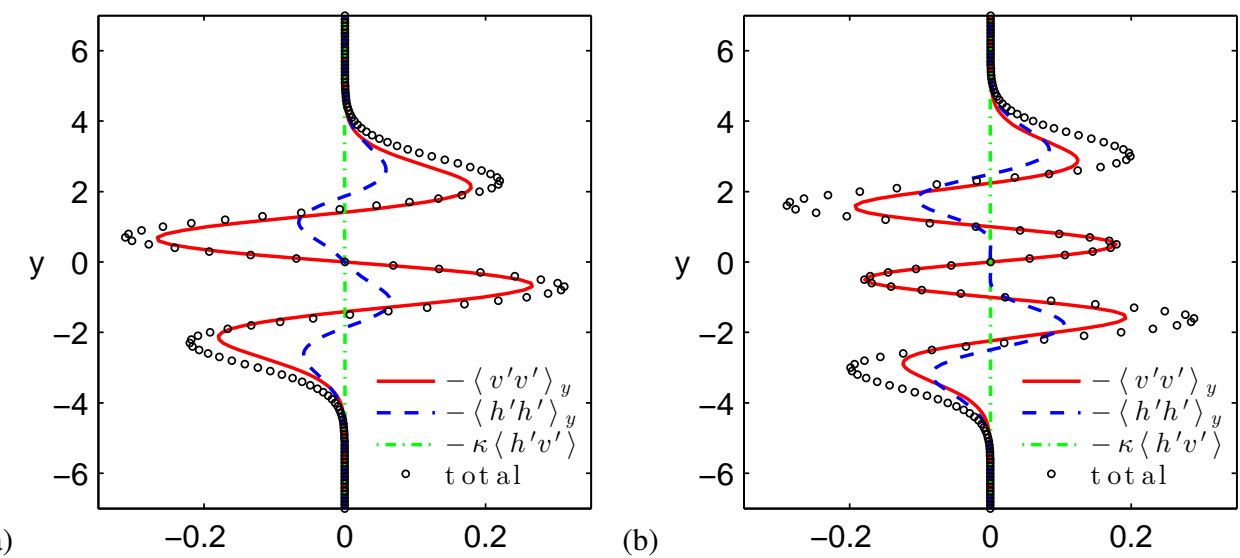

FIGURE 11. The effective meridional forcing terms $-\frac{1}{2}\left\langle h^{\prime 2}\right\rangle_{y},-\left\langle v^{\prime} v^{\prime}\right\rangle_{y}$ and $-\kappa\left\langle h^{\prime} u^{\prime}\right\rangle$ on the right hand side of (5.6c), and their sum, for dissipation solely by radiative relaxation $(\kappa=0.001, \gamma=0)$. All terms are calculated for $k=1, a=1$ for scaling, and either (a) $n=1$ or (b) $n=2$. The bolus velocity term $-\kappa\left\langle h^{\prime} u^{\prime}\right\rangle$ is much smaller than the other two terms, which are not proportional to $\kappa$.

mass conservation equation with no source terms. However, the combination $h^{2}(1+\theta)$ appears in both the pressure gradient in (5.17) and the radiative relaxation term in (5.18). We may reduce this expression to a quadratic product by writing

$$
h^{2} \theta=h \theta+h^{\prime} \theta^{\prime}+\mathcal{O}\left(a^{3}\right) .
$$


The zonal mean of the temperature equation may thus be written as

$$
\langle\theta\rangle_{t}^{*}+\kappa\left(\langle\theta\rangle^{*}+\langle h\rangle-1\right)=-\left\langle v^{\prime} \theta^{\prime}\right\rangle_{y}-\kappa\left(\left\langle h^{\prime} \theta^{\prime}\right\rangle+\left\langle h^{\prime 2}\right\rangle\right) .
$$

As before for the velocity components, we use $\langle\theta\rangle^{*}$ instead of $\langle\theta\rangle$ to absorb a contribution from $\left\langle\theta^{\prime} h^{\prime}\right\rangle$ in the time derivative. As $\theta=\mathcal{O}(a)$ under our decomposition, we may neglect factors of $\langle h\rangle=1+\mathcal{O}\left(a^{2}\right)$ that we could not neglect for $\langle\Theta\rangle^{*}$. Similarly, the zonal mean of the meridional momentum equation is

$$
\langle v\rangle_{t}^{*}+\frac{1}{2} y\langle u\rangle^{*}+\gamma\langle v\rangle^{*}+\langle h\rangle_{y}+\frac{1}{2}\langle\theta\rangle_{y}^{*}=-\left\langle v^{\prime} v^{\prime}\right\rangle_{y}-\frac{1}{2}\left\langle h^{\prime 2}\right\rangle_{y}-\frac{1}{2}\left\langle h^{\prime} \theta^{\prime}\right\rangle_{y},
$$

so we again have four equations for evolving $\langle h\rangle,\langle u\rangle^{*},\langle u\rangle^{*},\langle\theta\rangle^{*}$ with linear left hand sides. The right hand sides are again zonal means of quadratic products of the fluctuating quantities $h^{\prime}, u^{\prime}, v^{\prime}, \theta^{\prime}$.

The meridional velocity perturbation for an equatorial Rossby wave is again $v^{\prime}=\alpha \operatorname{Re}\{\hat{v}(y) \exp (\mathrm{i}(k x-\omega t))\}$, with $\hat{v}(y)$ defined by (4.3) and (4.10). The corresponding perturbations to the thickness, temperature, and zonal velocity take the same functional forms, with

$$
\begin{aligned}
& \hat{h}(y)=-\mathrm{i}\left(\frac{\mathrm{i} k \hat{u}+\mathrm{d} \hat{v} / \mathrm{d} y}{\omega}\right), \quad \hat{\Theta}(y)=-\mathrm{i}\left(\frac{\kappa \hat{h}}{\omega+\mathrm{i} \kappa}\right), \\
& \hat{u}(y)=\mathrm{i}\left(\frac{k(1+\mathrm{i} \kappa /(2 \omega)) \mathrm{d} \hat{v} / \mathrm{d} y-\frac{1}{2} y(\omega+\mathrm{i} \kappa) \hat{v}}{k^{2}(1+\mathrm{i} \kappa /(2 \omega))-(\omega+\mathrm{i} \gamma)(\omega+\mathrm{i} \kappa)}\right),
\end{aligned}
$$

for $\omega \neq 0$ and $\omega \neq-\mathrm{i} \kappa$. The vanishing denominator in the expression for $\hat{u}$ when $k^{2}=(\omega+\mathrm{i} \gamma)(\omega+\mathrm{i} \kappa) /(1+\mathrm{i} \kappa /(2 \omega))$ again gives the dispersion relation for the equatorially trapped Kelvin waves. We choose the normalisation constant $\alpha$ so that the disturbances $u^{\prime}, v^{\prime}, h^{\prime}$ and $\theta^{\prime}$ satisfy

$$
\frac{1}{2} \int\left\langle u^{\prime 2}\right\rangle+\left\langle v^{\prime 2}\right\rangle+\left\langle\left(h^{\prime}+\frac{1}{2} \theta^{\prime}\right)^{2}\right\rangle \mathrm{d} y=a^{2}
$$

The left hand side is the dimensionless quadratic approximation for small amplitude disturbances to the pseudoenergy

$$
\frac{1}{2} \int h\left(u^{2}+v^{2}\right)+\left(h \sqrt{\Theta}-h_{0} \sqrt{\Theta_{0}}\right)^{2} \mathrm{~d} x \mathrm{~d} y
$$

of finite amplitude disturbances from a rest state of constant depth $h_{0}$ and temperature $\Theta_{0}$ in the thermal shallow water equations (Ripa 1995; Røed 1997; Warneford \& Dellar 2013)

Figures 12 and 13 show the Reynolds stress convergences on the right hand side of (5.15). These all closely resemble those for the previous shallow water equations with radiative relaxation of the thickness. Thus the mean flow at the equator $(y=0)$ is almost identical to that for the previous model, provided $\kappa$ is rescaled by a factor of 2 . A perturbative analysis similar to that in $\S 4.1$ shows that the Reynolds stress $\left\langle u^{\prime} v^{\prime}\right\rangle$ for this model is proportional to $\kappa / 2-\gamma$ for small $\kappa$ and $\gamma$.

Figure 14 shows the forcing terms in the right hand side of the mean meridional momentum equation (5.21). The dominant terms, $-\left\langle v^{\prime} v^{\prime}\right\rangle_{y}$ and $-\frac{1}{2}\left\langle h^{\prime 2}\right\rangle_{y}$ are very similar to those in figure 11 for the shallow water model with relaxation of the thickness. The thermal term $-\frac{1}{2}\left\langle h^{\prime} \theta^{\prime}\right\rangle_{y}$ is much smaller. Finally, figure 15 shows the forcing terms in the right hand side of the mean temperature equation (5.20). These all vanish for dissipation solely by friction $(\kappa=0)$, since the temperature perturbation $\hat{\theta}$ given by (5.22) then vanishes, so they are only shown for $\kappa>0$ and $\gamma=0$. As for the meridional velocity, the contribution from $\left\langle h^{\prime} \theta^{\prime}\right\rangle$ is much smaller than the other contributions.

\subsection{Discussion}

Our simulation parameters imply a dimensionless radiative relaxation rate $\kappa=7.88 \times 10^{-3}$ roughly 23 times larger than our dimensionless frictional rate $\gamma=3.42 \times 10^{-4}$. In $\S 5.1$ we established that the Reynolds stress $\left\langle u^{\prime} v^{\prime}\right\rangle$ in the mean zonal momentum equation is proportional to $\kappa-\gamma$ (for thickness relaxation) or $\kappa / 2-\gamma$ (for temperature relaxation) for small relaxation and friction rates. For $\kappa \gg \gamma$, our theory thus suggests that $\langle u\rangle^{*}>0$ on the equator, consistent with the super-rotating equatorial jets seen in our simulations. Conversely, for the purely frictional case $(\kappa=0)$ our theory gives $\langle u\rangle^{*}<0$ on the equator, again consistent with the sub-rotating equatorial jet in our simulation. These conclusions hold for the two shallow water models that relax either the thickness or the temperature. The Reynolds stresses in the two models are very similar for small relaxation and friction rates, provided one rescales the radiative relaxation rate $\kappa$ in the thermal model by a factor of 2, as in figures 12 (a) and 13(a).

We have performed a similar analysis for decaying equatorially trapped Kelvin, Yanai, and inertia-gravity waves in the two shallow water models with radiative relaxation of the thickness or temperature. The effective zonal forces due to these waves are at least an order of magnitude smaller than those due to decaying Rossby waves of the same pseudoenergy. Thus, as expected from our global QG simulations, these other types of wave make no significant contribution to setting the direction of the equatorial jet.

Our thermal shallow water model conserves both mass and momentum in the absence of friction $(\gamma=0)$. This offers a simple demonstration that there is no inconsistency between our calculation of positive (eastward) zonal acceleration 

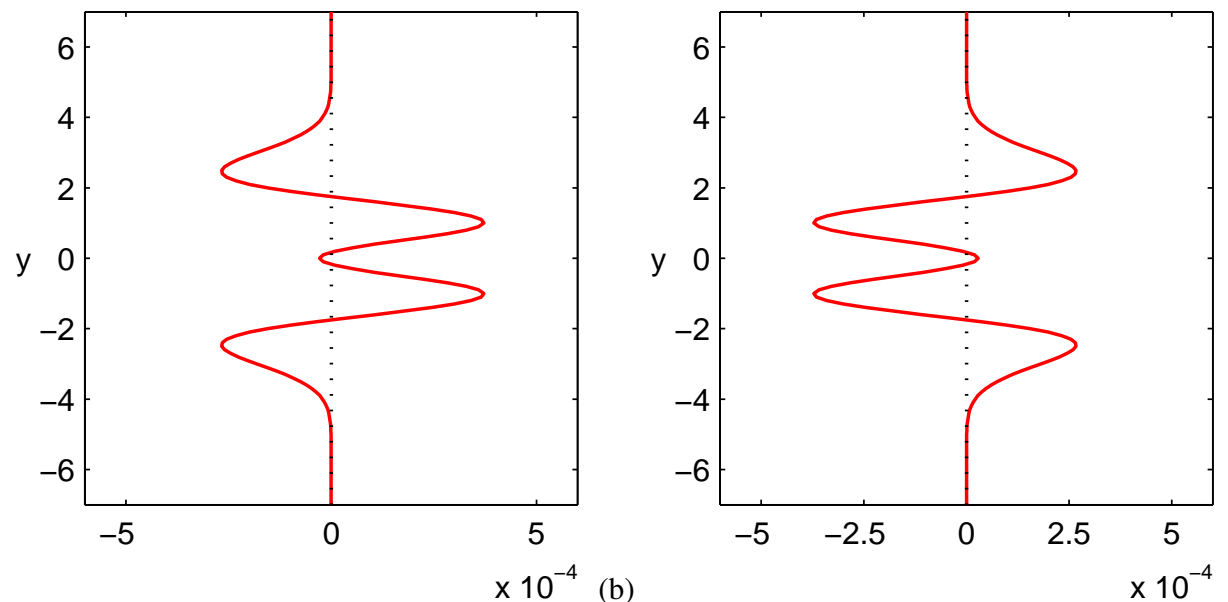

FIgURE 12. The effective zonal force $-\left\langle u^{\prime} v^{\prime}\right\rangle_{y}$ on the right hand side of (5.15) for $k=1, n=1$, and $a=1$ for scaling. Panel (a) shows dissipation solely by radiative relaxation $(\kappa=0.002, \gamma=0)$ and panel (b) shows dissipation solely by friction $(\kappa=0, \gamma=0.001)$. The zero line is shown dotted. The ratio of $\kappa$ to $\gamma$ reflects the symmetry of the dispersion relation in $\S 4.2$.

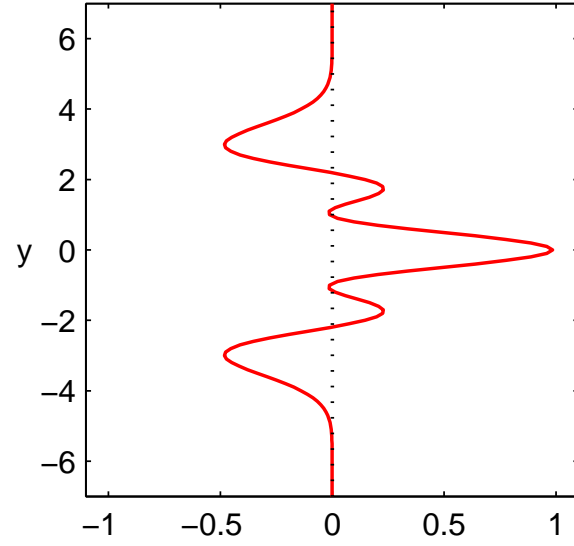

(a)

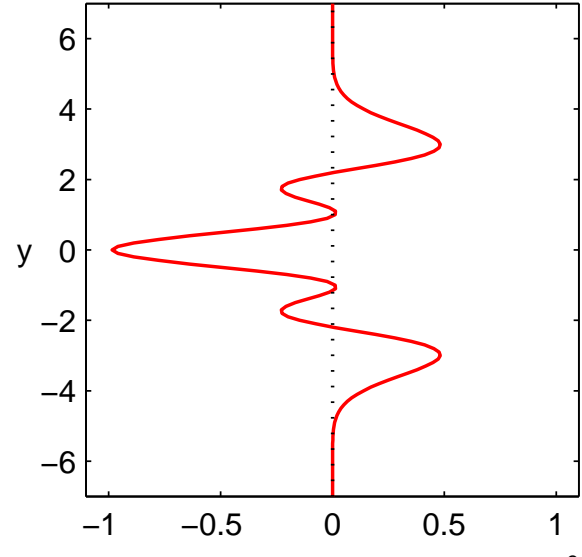

(b)

FIGURE 13. The effective zonal force $-\left\langle u^{\prime} v^{\prime}\right\rangle_{y}$ on the right hand side of (5.15) for $k=1, n=2$, and $a=1$ for scaling. Panel (a) shows dissipation solely by radiative relaxation $(\kappa=0.002, \gamma=0)$ and panel (b) shows dissipation solely by friction $(\kappa=0, \gamma=0.001)$. The zero line is shown dotted. The ratio of $\kappa$ to $\gamma$ reflects the symmetry of the dispersion relation in $\S 4.2$.

(a)

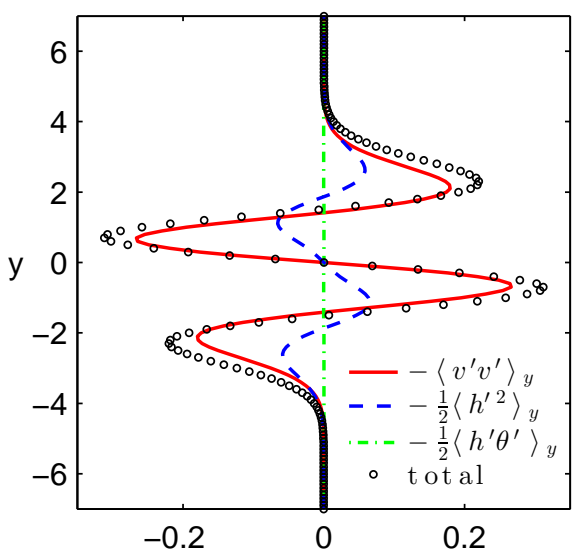

(b)

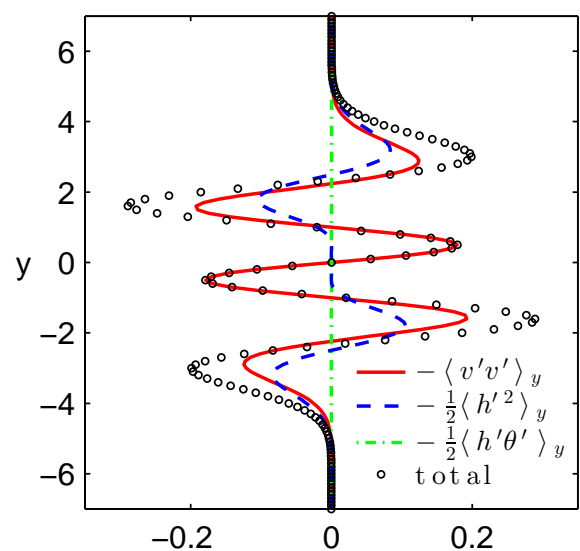

FIGURE 14. The effective meridional forcing terms $-\left\langle v^{\prime} v^{\prime}\right\rangle_{y},-\frac{1}{2}\left\langle h^{\prime 2}\right\rangle_{y},-\frac{1}{2}\left\langle h^{\prime} \theta^{\prime}\right\rangle_{y}$ on the right hand side of (5.21), and their sum, for dissipation solely by radiative relaxation $(\kappa=0.002, \gamma=0)$. All terms are calculated for $k=1, a=1$ for scaling, and either (a) $n=1$ or (b) $n=2$.

at the equator due to radiative dissipation of Rossby waves and the arguments reviewed in $\S 2$ that Rossby waves carry westward pseudomomentum. The evolution equation for $u$ may be rewritten in conservation form as

$$
\partial_{t}\left(h\left(u-y^{2} / 4\right)\right)+\partial_{x}\left(\left(h u\left(u-y^{2} / 4\right)+\Theta h^{2} / 2\right)+\partial_{y}\left(h v\left(u-y^{2} / 4\right)\right)=0 .\right.
$$

The combination $u-y^{2} / 4$ corresponds to the zonal velocity seen in an inertial frame, such that $\partial_{x} v-\partial_{y}\left(u-y^{2} / 4\right)=$ 

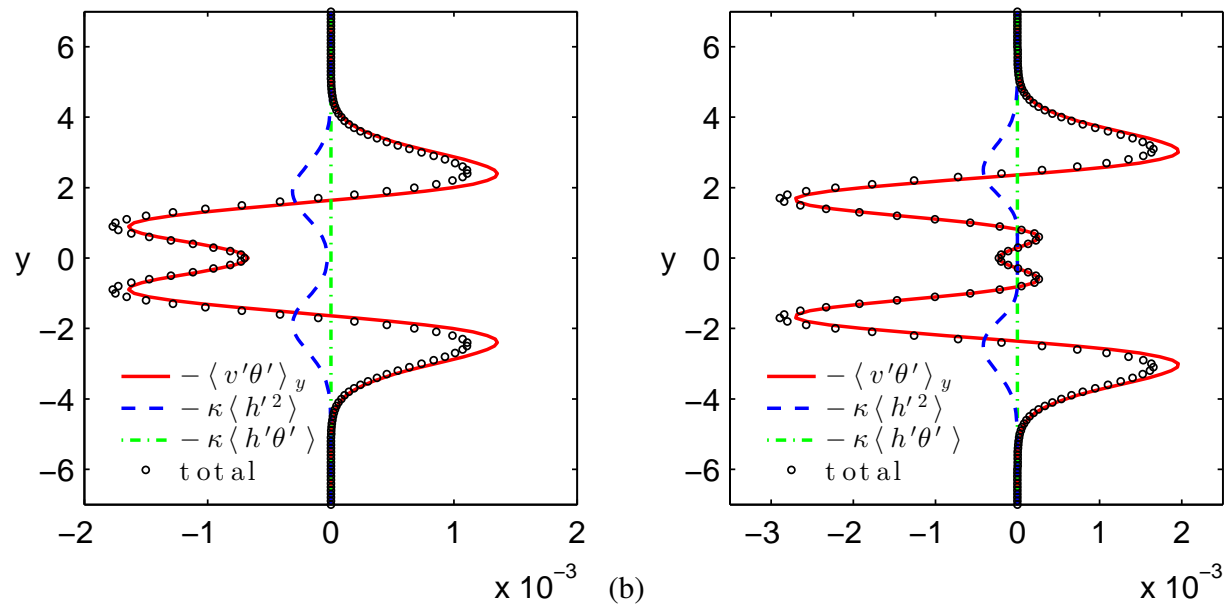

FIGURE 15. The effective thermal terms $-\left\langle v^{\prime} \theta^{\prime}\right\rangle_{y},-\kappa\left\langle h^{\prime} \theta^{\prime}\right\rangle,-\kappa\left\langle h^{\prime 2}\right\rangle$ on the right hand side of (5.20), and their sum, for dissipation solely by radiative relaxation $(\kappa=0.002, \gamma=0)$. All terms are calculated for $k=1, a=1$ for scaling, and either (a) $n=1$ or (b) $n=2$.

$y / 2+\partial_{x} v-\partial_{y} u$ is the absolute vorticity (Ripa 1993). The combination $h\left(u-y^{2} / 4\right)$, sometimes called absolute zonal momentum, is the beta-plane analogue of azimuthal angular momentum in spherical geometry.

The zonal mean of $(5.25)$ is

$$
\partial_{t}\left\langle h\left(u-y^{2} / 4\right)\right\rangle+\partial_{y}\left\langle h v\left(u-y^{2} / 4\right)\right\rangle=0
$$

which becomes

$$
\partial_{t}\left(\langle u\rangle^{*}-\frac{1}{4} y^{2}\langle h\rangle\right)+\partial_{y}\left(\left\langle u^{\prime} v^{\prime}\right\rangle-\frac{1}{4} y^{2}\langle h v\rangle\right)=0
$$

correct to $\mathcal{O}\left(a^{2}\right)$. Integrating (5.27) over $y$ implies that the global absolute zonal momentum

$$
\mathcal{M}=\int_{-\infty}^{\infty} \mathrm{d} y\langle u\rangle^{*}-\frac{1}{4} y^{2}(\langle h\rangle-1)
$$

is constant in time. We have added the time-independent term $y^{2} / 4$ to the integrand defining $\mathcal{M}$ so that this integral converges when $\langle h\rangle \rightarrow 1$ as $y \rightarrow \pm \infty$. Evaluating $\mathcal{M}$ at $t=0$ for the equatorially trapped Rossby wave solution from $\S 4.2$ for which $\langle h\rangle=1$ and $\langle u\rangle=0$ initially, we obtain

$$
\left.\mathcal{M}\right|_{t=0}=\int_{-\infty}^{\infty} \mathrm{d} y \frac{1}{2} \operatorname{Re}\left\{\hat{h}(y) \hat{u}(y)^{*}\right\}<0,
$$

where $\hat{h}(y)$ and $\hat{u}(y)$ are defined in (5.22), and $\hat{v}(y)$ is defined by (4.3) and (4.10). This negative sign agrees with the arguments reviewed in $\S 2$ that Rossby waves carry westward pseudomomentum.

The mass-weighted mean zonal velocity $\langle u\rangle^{*}$ generated by the decaying Rossby wave may be positive (eastward) at the equator, but the meridional integral of $\langle u\rangle^{*}-\frac{1}{4} y^{2}(\langle h\rangle-1)$ that defines $\mathcal{M}$ is still negative (westward). This is all that is required to reconcile conservation of zonal angular momentum with the requirement that Rossby waves should carry westward zonal pseudomomentum, as established concretely by (5.29).

\section{Conclusions}

Numerical simulations of shallow water equations on a sphere with isotropic random forcing reliably produce a mix of coherent vortices and alternating zonal jets. These simulations typically include Rayleigh friction to absorb the gradual inverse cascade of energy past the Rhines scale, since the zonal jets would otherwise break up into domainsized coherent vortices after very long times. Such simulations invariably produce sub-rotating equatorial jets when run with Jovian parameters (Vasavada \& Showman 2005). However, simulations that model radiative effects by relaxing the thickness field produce a super-rotating equatorial jet (Scott \& Polvani 2007, 2008), as do simulations of the thermal shallow water equations with a radiative relaxation term in the temperature equation (see $\S 3$ and Warneford \& Dellar 2014).

We have provided a possible explanation for the different directions of the equatorial jets in different shallow water models with dissipation by Rayleigh friction or Newtonian radiative relaxation. We formulated evolution equations for the zonal mean thickness $\langle h\rangle$, and the thickness-weighted zonal mean quantities $\langle u\rangle^{*},\langle v\rangle^{*},\langle\theta\rangle^{*}$. These equations contain effective forces due to zonal means of products of fluctuations, such as the Reynolds stress convergence $\left\langle u^{\prime} v^{\prime}\right\rangle_{y}$ in the evolution equation for $\langle v\rangle^{*}$. We evaluated these effective forces for an equatorially trapped Rossby waves superimposed on a background rest state on an equatorial beta plane. Dissipation changes the usual phase relations between the perturbations $u^{\prime}, v^{\prime}, h^{\prime}$, and permits a nonzero Reynolds stress $\left\langle u^{\prime} v^{\prime}\right\rangle$ that transports zonal momentum in the meridional 
direction. This Reynolds stress is proportional to $\kappa-\gamma$ for the shallow water model with relaxation of the thickness, and to $\kappa / 2-\gamma$ for the thermal shallow water model with relaxation of the temperature. The Reynolds stress divergence $\left\langle u^{\prime} v^{\prime}\right\rangle_{y}$ is the sole forcing term in the equation for $\langle u\rangle_{t}^{*}$ in the momentum-conserving shallow water models, and the larger forcing term in the Scott \& Polvani $(2007,2008)$ model with radiative relaxation of both thickness and momentum. In all cases, Newtonian radiative relaxation produces a Reynolds stress with the opposite sign to that due to Rayleigh friction. This suffices to determine that the zonal mean flow $\langle u\rangle^{*}$ on the equator created by the decaying Rossby wave with $n=2$ is eastward (super-rotating) for dissipation by Newtonian radiative relaxation, and westward (sub-rotating) for dissipation by Rayleigh friction.

Our work relies upon several major simplifying assumptions. We considered trapped equatorial Rossby waves on a background rest state, which allowed us to use the known analytical expressions for these waves in terms of Hermite polynomials. A more complete theory would calculate the Rossby wave spectrum supported by a zonally symmetric background flow. Secondly, we only calculated the effective forces in the evolution equations for the thickness-weighted zonal mean quantities $\langle u\rangle^{*},\langle v\rangle^{*},\langle\tilde{\theta}\rangle^{*}$. This is only sufficient to determine the evolution of $\langle u\rangle^{*}$ at the equator. Elsewhere, one would need to solve the system of three or four coupled equations to determine the Coriolis contribution to $\langle u\rangle^{*}$ from $\langle v\rangle^{*}$. However, it is worth noting that the latitude-dependence of the Reynolds stress divergence $\left\langle u^{\prime} v^{\prime}\right\rangle_{y}$ for the $n=1$ equatorially trapped Rossby wave, with two local maxima around $y= \pm 1$ in figures 9(a) and 12(a), is suggestive of the presence of two "horns", peaks of maximal eastward velocity, at latitudes around $\pm 7^{\circ}$ near the edges of the broad Jovian equatorial jet, and a slightly smaller velocity precisely on the equator (see figure 1).

Finally, we assumed that the waves are excited by sources outside the equatorial region, and neglected the effect of this excitation on the zonal mean flow. Some support for this second assumption is offered by simulations of the quasilinear version of the Scott \& Polvani (2007, 2008) model in spherical geometry by Saito \& Ishioka (2015) which show that the zonal mean zonal acceleration due to the ensemble of Rossby waves excited by random forcing of the wavenumbers in the annulus described in $\S 3$, and decaying by either radiative relaxation or friction, produce an equatorial jet in the direction predicted by our arguments, and with an amplitude consistent at early times with their fully nonlinear simulations. They also gave an elegant argument to determine the direction of the zonal acceleration from the tilting of the constant phase lines in the separable Rossby wave solutions, as previously demonstrated by Andrews \& McIntyre (1976b) for stratified fluids, and later adapted to shallow water models by Mofjeld (1981) and Yamagata \& Philander (1985). Although our work falls short of a full wave-mean flow interaction theory, we believe it offers at least a step towards a theoretical explanation of the origins of equatorial super- or sub-rotation in shallow water simulations of Jovian atmospheres.

We thank Michael McIntyre for very detailed and stimulating referee reports that greatly improved our manuscript, and Rick Salmon and Ted Johnson for useful conversations. This work was supported by the Engineering and Physical Sciences Research Council through a Doctoral Training Grant award to E.S.W. and an Advanced Research Fellowship to P.J.D. [grant number EP/E054625/1]. The computations employed the University of Oxford's Advanced Research Computing facilities (Richards 2015), and the Emerald GPU-accelerated High Performance Computer made available by the Science and Engineering South Consortium in partnership with the Science and Technology Facilities Council's Rutherford-Appleton Laboratory.

\section{REFERENCES}

Andrews, D. \& MCIntyre, M. 1976a Planetary waves in horizontal and vertical shear: The generalized Eliassen-Palm relation and the mean zonal acceleration. J. Atmos. Sci. 33, 2031-2048.

Andrews, D. \& MCIntyre, M. 1976b Planetary waves in horizontal and vertical shear: Asymptotic theory for equatorial waves in weak shear. J. Atmos. Sci. 33, 2049-2053.

Andrews, D. G. \& MCInTYRe, M. E. 1978 Generalized Eliassen-Palm and Charney-Drazin theorems for waves on axisymmetric mean flows in compressible atmospheres. J. Atmos. Sci. 35, 175-185.

BeEbe, R. 1994 Characteristic zonal winds and long-lived vortices in the atmospheres of the outer planets. Chaos 4, 113-122.

BÜHLER, O. 2000 On the vorticity transport due to dissipating or breaking waves in shallow-water flow. J. Fluid Mech. 407, 235-263.

BüHLER, O. 2014 Waves and Mean Flows, 2nd edn. Cambridge: Cambridge University Press.

Charney, J. G. 1949 On a physical basis for numerical prediction of large-scale motions in the atmosphere. J. Atmos. Sci. 6, $372-385$

Cho, J. Y.-K. \& Polvani, L. M. $1996 a$ The emergence of jets and vortices in freely evolving, shallow-water turbulence on a sphere. Phys. Fluids 8, 1531-1552.

Cho, J. Y.-K. \& Polvani, L. M. $1996 b$ The morphogenesis of bands and zonal winds in the atmospheres on the giant outer planets. Science 273, 335-337.

DALEY, R. 1983 Linear non-divergent mass-wind laws on the sphere. Tellus A 35A, 17-27.

Dickinson, R. E. 1969 Theory of planetary wave-zonal flow interaction. J. Atmos. Sci. 26, 73-81.

Dowling, T. E. 1995 Estimate of Jupiter's deep zonal-wind profile from Shoemaker-Levy 9 data and Arnol'd's second stability criterion. Icarus 117, 439-442.

Dowling, T. E. \& Ingersoll, A. P. 1989 Jupiter's Great Red Spot as a shallow water system. J. Atmos. Sci. 46, $3256-3278$.

GILl, A. E. 1982 Atmosphere Ocean Dynamics. New York: Academic Press. 
GreEN, J. S. A. 1970 Transfer properties of the large-scale eddies and the general circulation of the atmosphere. Q. J. R. Meteorol. Soc. 96, 157-185.

Haynes, P. H. \& MCIntyre, M. E. 1990 On the conservation and impermeability theorems for potential vorticity. J. Atmos. Sci. 47, 2021-2031.

Held, I. M. 2000 The General Circulation of the Atmosphere: Superrotation. Lectures presented at the 2000 Geophysical Fluid Dynamics Summer Program, Woods Hole Oceanographic Institution, Woods Hole, MA, available from http: //www. whoi . edu/page. do?pid=13076.

HIRST, A. C. 1986 Unstable and damped equatorial modes in simple coupled ocean-atmosphere models. J. Atmos. Sci. 43, 606-632.

HoU, T. Y. \& LI, R. 2007 Computing nearly singular solutions using pseudo-spectral methods. J. Comp. Phys. 226, 379-397.

HupCA, I. O., FAlCOU, J., GRIGORI, L. \& STOMPOR, R. 2012 Spherical harmonic transform with GPUs. Lecture Notes in Computer Science 7155, 355-366.

Iacono, R., Struglia, M. V. \& Ronchi, C. $1999 a$ Spontaneous formation of equatorial jets in freely decaying shallow water turbulence. Phys. Fluids 11, 1272-1274.

Iacono, R., Struglia, M. V., Ronchi, C. \& Nicastro, S. 1999b High-resolution simulations of freely decaying shallow-water turbulence on a rotating sphere. Il Nuovo Cimento C 22, 813-821.

INGERSOLL, A. P. 1990 Atmospheric dynamics of the outer planets. Science 248, 308-315.

Ingersoll, A. P., Dowling, T. E., Gierasch, P. J., Orton, G. S., Read, P. L., Sánchez-Lavega, A., Showman, A. P., Simon-Miller, A. A. \& VASAVAdA, A. R. 2007 Dynamics of Jupiter's atmosphere. In Jupiter: The Planet, Satellites and Magnetosphere (ed. F. Bagenal, T. E. Dowling \& W. B. McKinnon), pp. 105-128. Cambridge: Cambridge University Press.

JuCKeS, M. 1989 A shallow water model of the winter stratosphere. J. Atmos. Sci. 46, 2934-2956.

Khouider, B., Majda, A. J. \& Stechmann, S. N. 2013 Climate science in the tropics: waves, vortices and PDEs. Nonlinearity 26, R1-R68.

LAVOIE, R. L. 1972 A mesoscale numerical model of lake-effect storms. J. Atmos. Sci. 29, 1025-1040.

LIMAYE, S. S. 1986 Jupiter: New estimates of the mean zonal flow at the cloud level. Icarus 65, 335-352.

LiU, J. \& Schneider, T. 2010 Mechanisms of jet formation on the giant planets. J. Atmos. Sci. 67, 3652-3672.

MajdA, A. J. \& KLEIN, R. 2003 Systematic multiscale models for the tropics. J. Atmos. Sci. 60, 393-408.

MARCUS, P. S. 1988 Numerical simulation of Jupiter's Great Red Spot. Nature 331, 693-696.

Matsuno, T. 1966 Quasi-geostrophic motions in the equatorial area. J. Meteorol. Soc. Japan 44, 25-43.

MATSUNO, T. 1970 Vertical propagation of stationary planetary waves in the winter northern hemisphere. J. Atmos. Sci. 27, 871-883.

Matsuno, T. 1971 A dynamical model of the stratospheric sudden warming. J. Atmos. Sci. 28, 1479-1494.

MCCreary, J. P. 1985 Modeling equatorial ocean circulation. Annu. Rev. Fluid Mech. 17, 359-409.

McCreary, J. P., FukAmaChI, Y. \& KUndU, P. K. 1991 A numerical investigation of jets and eddies near an eastern ocean boundary. J. Geophys. Res. 96, 2515-2534.

MCCREARY, J. P. \& KUndu, P. K. 1988 A numerical investigation of the Somali Current during the Southwest Monsoon. J. Marine Res. 46, 25-58.

MCCREARY, J. P. \& YU, Z. 1992 Equatorial dynamics in a $2 \frac{1}{2}$-layer model. Prog. Oceanogr. 29, 61-132.

MCInTYRe, M. E. 1981 On the 'wave momentum' myth. J. Fluid Mech. 106, 331-347.

MCIntyre, M. E. \& Norton, W. A. 1990 Dissipative wave-mean interactions and the transport of vorticity or potential vorticity. J. Fluid Mech. 212, 403-435.

MofJELD, H. O. 1981 An analytic theory on how friction affects free internal waves in the equatorial waveguide. J. Phys. Oceanogr. 11, 1585-1590.

OвukHov, A. M. 1949 On the problem of the geostrophic wind. Izv. Akad. Nauk SSSR, Ser. Geograf. Geofiz. 13, $281-306$.

Philander, S. G. H., Yamagata, T. \& PaCAnowski, R. C. 1984 Unstable air-sea interactions in the tropics. J. Atmos. Sci. 41, 604-613.

Polvani, L. M., Waugh, D. W. \& Plumb, R. A. 1995 On the subtropical edge of the stratospheric surf zone. J. Atmos. Sci. 52, 1288-1309.

Porco, C. C., West, R. A., McEwen, A., Del Genio, A. D., Ingersoll, A. P., Thomas, P., Squyres, S., Dones, L., Murray, C. D., Johnson, T. V., Burns, J. A., Brahic, A., Neukum, G., VeVerka, J., Barbara, J. M., Denk, T., Evans, M., Ferrier, J. J., Geissler, P., Helfenstein, P., Roatsch, T., Throop, H., Tiscareno, M. \& Vasavada, A. R. 2003 Cassini imaging of Jupiter's atmosphere, satellites, and rings. Science 299, 1541-1547.

RHINeS, P. B. 1975 Waves and turbulence on a beta-plane. J. Fluid Mech. 69, 417-443.

RICHARDS, A. 2015 University of Oxford Advanced Research Computing. Zenodo document doi:10.5281/zenodo. 22558.

RIPA, P. 1993 Conservation laws for primitive equations models with inhomogeneous layers. Geophys. Astrophys. Fluid Dynam. 70, 85-111.

RIPA, P. 1995 On improving a one-layer ocean model with thermodynamics. J. Fluid Mech. 303, 169-201.

RIPA, P. $1996 a$ Low frequency approximation of a vertically averaged ocean model with thermodynamics. Rev. Mex. Fís. 41, 117135.

RIPA, P. $1996 b$ Linear waves in a one-layer ocean model with thermodynamics. J. Geophys. Res. 101, 1233-1245.

RøED, L. P. 1997 Energy diagnostics in a $1 \frac{1}{2}$-layer, nonisopycnic model. J. Phys. Oceanogr. 27, 1472-1476.

RøED, L. P. \& SHI, X. B. 1999 A numerical study of the dynamics and energetics of cool filaments, jets, and eddies off the Iberian Peninsula. J. Geophys. Res. 104, 29817-29841.

SAIto, I. \& IshiOKA, K. 2015 Mechanism for the formation of equatorial superrotation in forced shallow-water turbulence with Newtonian cooling. J. Atmos. Sci. 72, 1466-1483.

SCHNEIDER, T. \& LIU, J. 2009 Formation of jets and equatorial superrotation on Jupiter. J. Atmos. Sci. 66, 579-601.

SCHOPF, P. S. \& CANE, M. A. 1983 On equatorial dynamics, mixed layer physics and sea surface temperature. J. Phys. Oceanogr. 13, 917-935.

Schubert, W. H., TAft, R. K. \& Silvers, L. G. 2009 Shallow water quasi-geostrophic theory on the sphere. J. Adv. Model. Earth Syst. 1, 2. 
ScotT, R. K. \& Polvani, L. M. 2007 Forced-dissipative shallow-water turbulence on the sphere and the atmospheric circulation of the giant planets. J. Atmos. Sci. 64, 3158-3176.

Scott, R. K. \& Polvani, L. M. 2008 Equatorial superrotation in shallow atmospheres. Geophys. Res. Lett. 35 , L24202.

ShePherd, T. G. 1993 A unified theory of available potential energy. Atmosphere-Ocean 31, 1-26.

Showman, A. P. 2007 Numerical simulations of forced shallow-water turbulence: Effects of moist convection on the large-scale circulation of Jupiter and Saturn. J. Atmos. Sci. 64, 3132-3157.

SRINIVASAN, K. \& Young, W. R. 2012 Zonostrophic instability. J. Atmos. Sci. 69, 1633-1656.

SRINIVASAN, K. \& Young, W. R. 2014 Reynolds stress and eddy diffusivity of $\beta$-plane shear flows. J. Atmos. Sci. 71, $2169-2185$.

Thompson, R. O. R. Y. 1971 Why there is an intense Eastward current in the North Atlantic but not in the South Atlantic. J. Phys. Oceanogr. 1, 235-237.

ThUbURn, J. \& LAgneAU, V. 1999 Eulerian mean, contour integral, and finite-amplitude wave activity diagnostics applied to a single-layer model of the winter stratosphere. J. Atmos. Sci. 56, 689-710.

Vallis, G. K. 2006 Atmospheric and Oceanic Fluid Dynamics. Cambridge University Press.

Vasavada, A. R. \& Showman, A. P. 2005 Jovian atmospheric dynamics: an update after Galileo and Cassini. Rep. Prog. Phys. 68, 1935-1996.

VERKLEY, W. T. M. 2009 A balanced approximation of the one-layer shallow-water equations on a sphere. J. Atmos. Sci. 66, 17351748.

WAlterscheid, R. L., Brinkman, D. G. \& Schubert, G. 2000 Wave disturbances from the comet SL-9 impacts into Jupiter's atmosphere. Icarus 145, 140-146.

WARNEFORD, E. S. 2014 The thermal shallow water equations, their quasi-geostrophic limit, and equatorial superrotation in Jovian atmospheres. DPhil thesis, University of Oxford, http://ora.ox.ac.uk/objects/uuid: $6604 \mathrm{fcac}-\mathrm{afe} 6-4 \mathrm{abe}-8 \mathrm{a} 6 \mathrm{f}-6 \mathrm{a} 09 \mathrm{de} 4 \mathrm{f} 933 \mathrm{f}$.

WARnEFord, E. S. \& Dellar, P. J. 2013 The quasi-geostrophic theory of the thermal shallow water equations. J. Fluid Mech. 723, 374-403.

WARneford, E. S. \& Dellar, P. J. 2014 Thermal shallow water models of geostrophic turbulence in Jovian atmospheres. Phys. Fluids 26, 016603.

Williams, G. P. 1978 Planetary circulations: 1. Barotropic representation of Jovian and terrestrial turbulence. J. Atmos. Sci. 35, $1399-1426$.

Williams, G. P. \& Yamagata, T. 1984 Geostrophic regimes, intermediate solitary vortices and Jovian eddies. J. Atmos. Sci. 41, 453-478.

Yamagata, T. \& Philander, S. 1985 The role of damped equatorial waves in the oceanic response to winds. J. Oceanogr. Soc. Japan 41, 345-357. 